\title{
Kinetics and Morphological Evolution of Liquid Metal Dealloying
}

\author{
Ian McCue ${ }^{1}$, Bernard Gaskey ${ }^{1}$, Pierre-Antoine Geslin ${ }^{2,3}$, Alain Karma ${ }^{2}$, Jonah Erlebacher ${ }^{1 *}$
}

1 Department of Materials Science and Engineering, Johns Hopkins University, Baltimore, MD 21218, USA

2 Physics Department and Center for Interdisciplinary Research on Complex Systems, Northeastern University, Boston, Massachusetts 02115, USA

3 Institut Lumière Matière, UMR 5306 Université Lyon 1-CNRS, 69622 Villeurbanne cedex, France

* Corresponding author: Jonah.Erlebacher@jhu.edu

\begin{abstract}
Liquid metal dealloying (LMD) has recently emerged as a novel technique to fabricate bulk nanostructures using a bottom-up self-organization method, but the literature lacks fundamental studies of this kinetic process. In this work, we conduct an in-depth study of the kinetics and fundamental microstructure evolution mechanisms during LMD using Ti-Ta alloys immersed in molten $\mathrm{Cu}$ as a model system. We develop a model of LMD kinetics based on a quantitative characterization of the effects of key parameters in our system including alloy composition, dealloying duration, and dealloying temperature. This work demonstrates that the dealloying interface is at or near equilibrium during LMD, and that the rate-limiting step is the liquidstate diffusion of dissolving atoms away from the dealloying interface (diffusion-limited kinetics). The quantitative comparison between theoretically predicted and measured dealloying rates further reveals that convective transport and rejection of the dissolving element during coarsening of the structure also influence the dealloying kinetics.
\end{abstract}




\section{Introduction}

Liquid metal dealloying (LMD) has recently emerged as a facile technique to fabricate bulk nanoporous and nanocomposite materials for a variety of applications including electrolytic capacitors, Li-ion battery anodes, and high-strength structural materials [1-5]. Dealloying is a corrosion process in metallic systems where one or more components are selectively dissolved from an alloy [6]. Historically, dealloying has been restricted to electrochemical corrosion where the less noble component(s) are removed, but the concept is now generalized to any embodiment of liquid-mediated dissolution. In electrochemical dealloying, the dissolving component(s) are removed by applying a potential which is above their standard reduction potential but below the standard reduction potential of the remaining component(s) [7-9]. During dissolution the remaining component(s) diffuse along the metal/liquid interface, self-organizing into a topologically complex, high surface area porous material. Analogously, LMD relies on enthalpies of mixing to facilitate dissolution [10,11]. Specifically, the liquid metal is chosen to have a negative enthalpy of mixing with the dissolving component(s) and a positive enthalpy of mixing with the remaining component(s). The analogous critical potential in LMD is the concentration of the dissolving component in the liquid bath: the highest driving force for dealloying is when the concentration of the dissolving component in the liquid bath is zero, and there should be no driving force for dealloying when the liquid bath is saturated with the dissolved component.

While a majority of the work on this subject has focused on the characterization and properties of nanomaterials made by LMD, a recent phase field modeling study has shed light on fundamental mechanisms of structure formation [12]. This study also briefly addressed the kinetics of the dealloying process, but focused primarily on explaining the variety of topologically 
distinct morphologies formed during LMD as a function of alloy composition [12]. The goal of the present manuscript is to comprehensively characterize the dealloying kinetics and morphological evolution during LMD. We present an in-depth analysis of dealloying kinetics that examines the effects of diffusive transport, convective transport, and coarsening of the structure, all of which influence the dealloying rate. Our results provide a quantitative framework for optimizing the fabrication of nanocomposite and nanoporous materials.

For this study we chose our model LMD system of Ti-Ta alloys immersed in molten $\mathrm{Cu}$. Ti and Ta form a solid solution with a body centered cubic (bcc) symmetry across the entire composition range at all processing temperatures, Ta is immiscible with $\mathrm{Cu}$, and molten $\mathrm{Cu}$ has a high Ti solubility (approximately 75 at\% for the temperature range in this study). During dealloying, molten $\mathrm{Cu}$ selectively dissolves $\mathrm{Ti}$ out of the alloy while $\mathrm{Ta}$ diffuses along the metal/liquid interface and reorganizes into a porous bicontinuous network. Copper remains inside the pores and fills the dealloyed-phase volume as it penetrates further into the alloy, and a dense $\mathrm{Ta} / \mathrm{Cu}$ nanocomposite is formed upon cooling. A nanoporous structure can be excavated by chemically or electrochemically dissolving the $\mathrm{Cu}$ phase. The surface mobility of $\mathrm{Ta}$ at room temperature in an electrolyte is very low so the refractory phase remains undisturbed during chemical etching (as well as forming a passive oxide). Additionally, the Ti-Ta system is not limited to dealloying in molten $\mathrm{Cu}$, and later in this manuscript we briefly examine LMD of Ti-Ta alloys in molten baths containing $\mathrm{Cu}, \mathrm{Ag}$, and $\mathrm{Bi}$. 


\section{Experimental Methods}

Experiments were carried out by immersing Ti-Ta alloy samples into $\mathrm{Cu}$ baths; we varied the alloy composition, immersion time and reaction temperature to investigate how these parameters affected the dealloying depth, ligament size, and concentration profile in the liquid phase.

Ti-Ta alloys were prepared in-house by radio-frequency (RF) induction using an Ambrel Ekoheat $45 \mathrm{~kW}$ system by melting Ti (99.995 wt.\%), and Ta (99.95 wt.\%) pellets from Kurt Lesker in a water-cooled copper crucible from Arcast Inc. under flowing $\operatorname{Ar}(99.999$ wt.\%). After casting the ingots were annealed under flowing Ar (99.999 wt.\%) for approximately 10 hours and compositional homogeneity was confirmed using energy-dispersive X-ray spectroscopy (EDS). Master ingots of $\sim 30 \mathrm{~g}$ were made of the following compositions: $\mathrm{Ti}_{70} \mathrm{Ta}_{30}, \mathrm{Ti}_{65} \mathrm{Ta}_{35}, \mathrm{Ti}_{55} \mathrm{Ta}_{45}$, $\mathrm{Ti}_{40} \mathrm{Ta}_{60}, \mathrm{Ti}_{30} \mathrm{Ta}_{70}$, and $\mathrm{Ti}_{20} \mathrm{Ta}_{80}$. Approximately $1 \mathrm{~g}$ samples (roughly 6 × 2 × $10 \mathrm{~mm}$ ) were cut for LMD from the master ingots using wire electrical discharge machining (wire EDM). After cutting, the samples were lightly sanded with 600 grit to remove the recast layer from wire EDM and Ta wires were spot-welded onto one end of each sample for the immersion experiments.

Prior to immersion, a $40 \mathrm{~g}$ molten $\mathrm{Cu}(99.99 \mathrm{wt} . \%)$ bath was prepared [for each sample] in a high-purity alumina crucible cast in-house with materials from Cotronics at a preset temperature $\left(1160,1240,1305\right.$, or $\left.1360{ }^{\circ} \mathrm{C}\right)$. Temperature measurements were made using an infrared camera set to the emissivity of molten $\mathrm{Cu}$. Prior to immersion, the sample was preheated by bringing it inside the RF coils -without touching the bath - to avoid thermal gradients between the sample and the bath. The sample was then immersed into molten $\mathrm{Cu}$ for a fixed time (between 10 and 120 seconds) and then removed from the bath, cooling the sample and solidifying the $\mathrm{Cu}$ inside the pores, halting the dealloying process. 
Cross sections of the nanocomposites were mounted, polished, and characterized using a JEOL scanning electron microscope (SEM) in order to determine the average ligament size and measure the dealloying depth as a function of time, temperature and composition. ImageJ was used to measure dealloying depth and average ligament sizes. The dealloying depth was determined by averaging 40 measurements taken from four images, which were from different regions of the sample. Error! Reference source not found. shows a panel of SEM images with typical cross sections (here, of Ti55 Ta45 dealloyed for 20 seconds at four different temperatures), illustrating our measurements of dealloying depth. The average ligament size was determined by averaging over 20 measurements for a given depth. We included both the ligament diameters and nodes in our measurements of the ligament size. Additionally, EDS was used to determine the composition of each phase as a function of depth. The composition for a given depth was determined by averaging over an area with a $1-5 \mu \mathrm{m}$ diameter.

\section{Results and Discussion}

\subsection{Dealloying Depth Data}

Error! Reference source not found. shows raw data for the dealloying depth as a function of time for three different compositions and four different temperatures. Error bars are not included, but all depth measurements had a standard deviation below $30 \mu \mathrm{m}$ and the majority had a standard deviation below $15 \mu \mathrm{m}$. Three obvious trends are apparent: the dealloying depth increases with time and temperature, and decreases with increasing Ta composition. The data also appears to follow a power-law relationship with time. We now introduce a dissolution model to interpret our data and understand the dealloying depth's functional dependence on time and temperature. 


\subsection{Dissolution Model}

Previous work on LMD systems have suggested the reaction is diffusion-limited and dependent on diffusion of the dissolving component (here, Ti) away from the dealloying interface [11,12], but have not introduced and rigorously tested a quantitative model. The concept of LMD in nobel metal systems was first introduced, and briefly explored, in 1959 by Harrison and Wagner who had also performed some of the initial work on electrochemical dealloying [13]. In this work they introduced a similar analytical model to the one described below, however their approach was heavily approximated because they were unable to measured concentration profiles and did not examine multiple temperatures. Consistent with previous work, here we assume that the dealloying kinetics are limited by diffusion of the dealloyed element in the liquid metal and further validate this hypothesis by quantitative comparison of theoretical predictions based on this assumption and experiments. Before we can introduce a dissolution model, however, we need to address two key points.

First, we need to assess the $\mathrm{Ti}$ concentration in the $\mathrm{Cu}$ phase at the dealloying interface. Specifically, we need to establish that the interface is at (or near) equilibrium during dealloying. This requires a direct measurement of the Ti concentration profile in the Cu phase in the dealloyed region, which we were able to accomplish because the $\mathrm{Cu}$ remains inside the pores and solidifies after dealloying. It is readily apparent in Error! Reference source not found. that the $\mathrm{Ti}$ concentration at the interface is unique for a given parent alloy composition, $\mathrm{Ti}_{\mathrm{x}} \mathrm{Ta}_{1-\mathrm{x}}$. This suggests that the interface is in chemical equilibrium and that the Ta composition at the interface has an influence on the equilibrium concentration, governed by the Ti-Ta-Cu ternary diagram and not the Ti-Cu binary diagram. Additionally, Error! Reference source not found. illustrates that the interface concentration is relatively constant across the timescales 
and temperature range we are studying; for both parameters we did not observe a significant difference outside the error of our measurement ( \pm 5 at.\%). The concentration values in Error!

\section{Reference source not found. do not approach}

Second, we need to address the dimensionality of this kinetic problem. In principle, the dissolving element is able to diffuse in three dimensions, but we are able to neglect lateral transport because the thickness of the diffusive boundary layer (of order $100 \mu \mathrm{m}$ ) - where the concentration of the dissolving element varies appreciably - is much larger than the pore size (of order $1 \mu \mathrm{m}$ ). As a result the LMD process can be simplified to a one-dimensional Stefan problem, the general class of free boundary problems where a phase boundary can move with time through a diffusionlimited process [14]. Since diffusion in the parent alloy is very slow at these temperatures, the velocity of the dealloying front is given by the flux of the dealloyed element towards the liquid (Stefan condition). This construction allows us to solve for the position of the dealloying interface and the concentration profile in the liquid within the dealloyed region as functions of time, which are central to understanding how to fabricate nanomaterials using this process.

Error! Reference source not found. illustrates LMD as a one-dimensional Stefan problem. Consider an isothermal system containing an infinite liquid bath of $\mathrm{Cu}$ and a semi-infinite block of Ti-Ta where $x_{i}(t)$ is the position of the interface between the two phases. When $t>0$, the $\mathrm{Cu}$ phase dissolves Ti from the Ti-Ta alloy, moving the interface into the solid. As the interface moves, the liquid phase collects the dissolved $\mathrm{Ti}$, and the rate-limiting process is Ti diffusion away from the interface. If the rate-limiting process were interface-limited we would expect the $\mathrm{Ti}$ concentration profile inside the dealloyed region to remain constant, and the dealloying velocity would remain constant $[15,16]$. However, we show below that the Ti gradient decreases over longer dealloying times, leading to a decrease in the dealloying velocity with increasing time. A 
recent study of dealloying in Mg-Cd alloys [17] revealed that lattice diffusion can play a role in dealloying (transport of the dissolving component from the bulk material to the dealloying interface) and lead to a dendritic void morphology. This requires the initial alloy composition to be below the parting limit, see Section 3.5, however the three alloys we chose for this study sit above this value. Furthermore, the interdiffusivity in Ti-Ta is several orders of magnitude smaller than the liquid-state diffusivity of $\mathrm{Ti}$ in the $\mathrm{Cu}$ melt, and does not appear to affect morphological evolution when the Ti composition is at least 40 at\%. To compare, at $1200{ }^{\circ} \mathrm{C}$ $D_{S}^{T i \square} \square a 10^{-9} \mathrm{~cm}^{2} s^{-1}$ and $D_{L}^{T i \rightarrow C u} \square 10^{-5} \mathrm{~cm}^{2} s^{-1}[18,19]$.

The concentration of $\mathrm{Ti}$ in the liquid phase, $c(x, t)$, follows the diffusion equation:

$$
\partial_{t} c=D_{L} \partial_{x}^{2} c
$$

where $D_{L}$ is the diffusivity of Ti in liquid $\mathrm{Cu}$. The Stefan condition at the moving boundary,

$$
\begin{aligned}
x= & x_{i}(t), \text { is } \\
& v_{i}\left(c_{S}^{T i}-c_{L}^{T i}\right)=\left.D_{L} \frac{\partial c}{\partial x}\right|_{i},
\end{aligned}
$$

where $v_{i}$ is the interface velocity, $c_{S}^{T i}$ is the concentration of Ti in the parent alloy at the interface, and $c\left(x_{i}(t), t\right)=c_{L}^{T i}$ is the concentration of Ti in the liquid at the interface. The latter is assumed to remain constant in time, consistent with the physical picture that dissolution is slow enough for the solid-liquid interface to be in quasi-local chemical equilibrium [12] and experimental measurements. The Ti concentration at the interface provides one of the boundary conditions for the concentration field $c(x)$, but the boundary conditions for the $\mathrm{Cu}$ bath is not as obvious. The most simple and natural boundary condition is to consider an infinite bath of liquid $\mathrm{Cu}$ into which Ti can diffuse; this can be translated mathematically into the boundary condition of Eq. (3). 
However, the actual dealloying experiments use RF induction to heat the liquid bath, which induces convection currents. Outside of the dealloyed region, i.e. $x>0$, it is reasonable to assume that the convection currents lead to a complete mixing of the dissolved $\mathrm{Ti}$ and the entirety of the $\mathrm{Cu}$ bath. On the other hand, in the dealloyed region, the finely structured solid ligaments are obstacles for these convective currents, and the Ti concentration of the liquid in the dealloyed region can be considered to follow purely diffusive dynamics ${ }^{1}$.

These assumptions translate into the boundary condition Eq. (4). As a result, we have two different boundary conditions for the $\mathrm{Cu}$ bath depending on whether or not we account for convection currents:

$$
\begin{aligned}
& c(x=+\infty, t)=c_{B}^{T i}, \\
& c(x \geq 0, t)=c_{B}^{T i},
\end{aligned}
$$

where $c_{B}^{T i}$ is the $\mathrm{Ti}$ concentration in the $\mathrm{Cu}$ bath. The bath composition is assumed to remain constant during the experiment - the $\mathrm{Cu}$ bath is 40 times greater in mass than the immersed sample. For our experiments the initial bath composition was always pure Cu because the driving force for dealloying is the largest when there is no pre-dissolved Ti. We can solve Eq. (1) and deduce the concentration profile, $c(x, t)$, by introducing $\Omega=\frac{c_{L}^{T i}-c_{B}^{T i}}{c_{S}^{T i}-c_{L}^{T i}}$ and the dimensionless Péclet number of the problem $p=x_{i} v_{i} / 2 D_{L}$. Here, $\Omega$ is the analogue to the supersaturation Stefan number in solidification problems. A more complete introduction of this diffusion problem can be found in Appendix A.1.

\footnotetext{
${ }^{1}$ It is possible to determine the degree of convection in our experiments by immersing samples into the molten melt and simultaneously rotating them to control the convection near the sample surface, and then and measuring the concentration profile (analogous to a rotating disc electrode experiment in electrochemistry). However, this is experimentally challenging and beyond the scope of this work.
} 
The general solution to Eq. (1), derived in Appendix A.2 and A.3, is given by Eqs. (A23) and (A30), repeated here:

$$
c(x, t)=c_{L}^{T i}+\left(c_{S}^{T i}-c_{L}^{T i}\right) \sqrt{p \pi} e^{p}\left(\operatorname{erf}\left(-\frac{x}{\sqrt{4 D t}}\right)-\operatorname{erf}(\sqrt{p})\right) .
$$

The difference between the two boundary conditions, Eqs. (3) and (4), is the relationship between $\Omega$ and $p$. The relationship for the simple diffusion boundary condition, Eq.(3), derived in Appendix A.2, is given by Eq. (A19), repeated here:

$$
\Omega=\sqrt{\pi p} e^{p}(1+\operatorname{erf}(\sqrt{p}))
$$

And the relationship for the boundary condition accounting for convection, Eq. (4), derived in Appendix A.3 is given by Eq. (A26), repeated here:

$$
\Omega=\sqrt{\pi p} e^{p}(\operatorname{erf}(\sqrt{p}))
$$

Eqs. (6) and (7) may appear to be nearly identical, however Error! Reference source not found. demonstrates that the resulting concentration profiles are quite different. Error! Reference source not found.b compares the two solutions along with experimental data using a literature value for the diffusivity of $\mathrm{Ti}$ in liquid $\mathrm{Cu}$ (discussed more below). It appears that the solution accounting for convection is more accurate, however the discrepancy at the edge of the sample suggests the convective currents affect the diffusion profile but do not mix perfectly the liquid exactly at the edge of the dealloyed structure. This would imply the real solution for the Ti concentration profile is between the two boundary conditions discussed above. For the sake of simplicity we use one solution for this manuscript, Eq. (A30). Using Eq. (7) we can re-express the concentration profile in a more compact form: 


$$
c(x, t)=\frac{c_{L}^{T i}-c_{B}^{T i}}{\operatorname{erf}(p)} \operatorname{erf}\left(-\frac{x}{\sqrt{4 D_{L} t}}\right)+c_{B}^{T i},
$$

where the Péclet number $p$ is computed by numerically inverting Eq. (7).

\subsection{Concentration Profiles}

In general, Eq. (8) fits our data well, with one caveat. As shown for example in Error! Reference source not found.b, our data deviates from our model near the original outer surface of our samples, where the raw data shows a greater concentration of Ti than predicted by the model. We speculated in the previous section that this could be a result of partial convective mixing, but this deviation could also be due to coarsening of the Ta-rich ligaments. Coarsening exposes new Ti to the melt as a secondary source, and is analogous to post-dealloying coarsening in electrochemical dealloying where the ligaments enrich in the noble component $[6,20]$. During coarsening, Ti inside the ligaments become exposed to the surface through Ta-mediated surface smoothening and genus-reduction events, and then dissolves into the liquid phase [21]. The Tarich ligaments can be considered finite point sources of $\mathrm{Ti}$, and this surface diffusion-mediated

process has an approximate $t^{1 / 4}$ dependence, which has a minor effect on the dealloying velocity (Section 3.4) [21,22]. Using Eq. (8), we performed a least squares fit - where $D_{L}$ is the only fitting parameter - on the experimental data for $1240{ }^{\circ} \mathrm{C}$ for each composition $\left(\mathrm{Ti}_{70} \mathrm{Ta}_{30}, \mathrm{Ti}_{55} \mathrm{Ta}_{45}\right.$, and $\mathrm{Ti}_{40} \mathrm{Ta}_{60}$ ), and obtained values for the diffusivities (Table 1) that are very close to literature values [19]. The literature values are from a series of experiments by Shurygin \& Shantarin where they measured Ti dissolution in molten $\mathrm{Cu}$ across the range $1100-1400{ }^{\circ} \mathrm{C}$ using a rotating $\mathrm{Ti}$ rod to overcome convective transfer and determine a true diffusion coefficient for $\mathrm{Ti}$ in molten $\mathrm{Cu}$. We excluded the portion where the experimental curves deviate from the model due to ligament 
coarsening during the fit. However, as shown in Error! Reference source not found., this region is quite small for the longer times and is not a significant source of error

Error! Reference source not found. illustrates that LMD is a relatively fast method to fabricate nanostructures, however it needs to be emphasized that this is an equilibrium process and dissimilar to a non-equilibrium process such as rapid solidification. It is clearly demonstrated in Error! Reference source not found. that the concentration at the dealloying interface is essentially constant for all dealloying times, as assumed in the dissolution model developed in section 3.2. If this were a non-equilibrium process the dealloying velocity would remain constant - instead of decreasing as it does here - and the Ti concentration at the interface would increase with increasing time.

\subsection{Dealloying Depth}

In the previous section we showed the experimental concentration profile can be fit using Eq. (8), and the diffusivity value was in close agreement with the literature value. Here we calculate the activation energy of the rate-limiting step in LMD and further confirm our hypothesis that the kinetics are controlled by $\mathrm{Ti}$ diffusion away from the dealloying interface. Following this assumption, we derived an expression for the dealloying depth in Appendix A.3, Eq. (A27), repeated here:

$$
x_{i}(t)=\sqrt{4 p D_{L} t}
$$

In the most general case, we can generate an Arrhenius plot for the dealloying depth by taking the natural logarithm of $x_{i}$ at a given dealloying time and assuming the diffusion in the liquid phase

is of the form $D_{L}=D_{0} e^{-E_{a} / k_{B} T}$. From this we can calculate $D_{0}$ and $E_{a}$ for each time, insets of Error! Reference source not found. and Table 1. Interestingly, the highest diffusivity values are 
calculated for shorter dealloying times and the lowest diffusivity value occurred at the longest time for each composition. We discuss this more in detail below, but we attribute this to coarsening, which we expect to have a larger effect at longer dealloying times. For this reason, the main plots of Error! Reference source not found. show $x_{i}$ versus $t^{\prime}=t * \exp \left(-E_{a} / k_{B} T\right)$ using $D_{0}$ and $E_{a}$ values averaged from the three earlier times for each composition. The average activation energies used in the data collapse of Error! Reference source not found. were: $0.57 \mathrm{eV}, 0.58 \mathrm{eV}$, and 1.16 eV for $\mathrm{Ti}_{70} \mathrm{Ta}_{30}, \mathrm{Ti}_{55} \mathrm{Ta}_{45}$, and $\mathrm{Ti}_{40} \mathrm{Ta}_{60}$, respectively. Comparing these activation energies to the literature value, $E_{T i \rightarrow C u}=0.715 \mathrm{eV}$, there is a slight discrepancy, however the activation energies for $\mathrm{Ti}_{70} \mathrm{Ta}_{30}$ and $\mathrm{Ti}_{55} \mathrm{Ta}_{45}$ at earlier times are closer. The average maximum diffusivities, $D_{0}$, used in the data collapse of Error! Reference source not found. were all within an order of magnitude of the literature value, Table 1.

There are two major reasons for the differences between our calculated $D_{0}$ and $E_{a}$ values and the literature value. First, we have simplified our analysis to only consider Ti diffusion away from the interface in a dilute limit. However, Ti concentrations in the dealloyed region reach high values where the dilute limit may not be valid. Thus, it is more accurate to say we are measuring a combined diffusivity of $\mathrm{Cu}$ and $\mathrm{Ti}$, which may be occurring at different rates. Second, as mentioned previously, we are not accounting for finite sources of $\mathrm{Ti}$ in the dealloyed region resulting from post-dealloying coarsening. The additional $\mathrm{Ti}$ added to the bath reduces the driving force for $\mathrm{Ti}$ diffusion away from the dealloying front, and a more rigorous form of Eq. (9) would include a second, albeit minor, term to account for Ti dissolution as a function of the coarsening behavior. Naturally, due to this slower time-dependence we expect shorter dealloying times (e.g. 10 seconds) to behave closer to the ideal case of Eq. (9) than longer times (e.g. 120 seconds). Our calculation 
of the activation energy is dependent on the power-law exponent of this process, and minor variations in the exponent can easily alter this value by several hundred meV. We will show in Section 3.6 that the activation energy for coarsening is comparable to the activation energy for $\mathrm{Ti}$ diffusion in molten $\mathrm{Cu}$, illustrating the difficulty in separating the contribution from these two processes in our current study. It is also worthwhile to mention that the activation energy associated with the composition $\mathrm{Ti}_{40} \mathrm{Ta}_{60}, E_{a}=1.16 \mathrm{eV}$, is a factor of two higher than the other two compositions, suggesting a different mechanism became the rate limiting step. This could either be a switch to attachment/detachment-limited regime as seen in electrochemical dealloying, or an effect of the morphology of the dealloyed structure, which will be discussed in Section 3.6.2.

Our final comment on the dealloying depth is the effect of grain boundaries, which can significantly alter the kinetics. Error! Reference source not found. is a cross-section of a $\mathrm{Ti}_{65} \mathrm{Ta}_{35}$ sample with an $\sim 50-100 \mu \mathrm{m}$ diameter grains (dealloying depth data not included in this work due to the ingot's small grain size) dealloyed at $1160{ }^{\circ} \mathrm{C}$ for 120 seconds. The dealloying interface is much rougher than the ones in Error! Reference source not found., making it difficult to measure an accurate dealloying depth. We speculate that this is the result of grain boundaries dealloying ahead of the interior of grains.

We observed grain boundaries are always dissolved first during LMD, replacing the solidsolid interface between adjacent grains with a solid-liquid-solid interface, Error! Reference source not found.b. Once liquid is in the grain boundary region, individual grains are subsequently dealloyed. Due to this asymmetric dissolution process grains can become detached and thick Tarich regions appear (Error! Reference source not found.). Obviously, it is not accurate to call these regions grain boundaries because the solid-solid interface is destroyed during dealloying, but we will use this name out of convenience. Dealloying is slower in regions where grain boundaries 
are perpendicular to the dealloying direction, and we think it is reasonable to conclude the boundaries impede Ti diffusing away from the dealloying interface. For this reason we made sure that all of the starting alloys quantitatively analyzed in this study were sufficiently annealed, and most samples had an average grain size of $500 \mathrm{um}$ in order to avoid grain boundaries affecting our measurements. We caution future work to be aware that boundaries can alter the kinetics, and it is desirable to have as large grains as possible to dealloy to large depths.

\subsection{Parting Limit}

We will briefly discuss the parting limit in liquid metal dealloying, which has been extensively studied in electrochemical dealloying due to its relevance in corrosion and passivation kinetics [23-25]. The parting limit, or percolation threshold $p_{c}$, is the critical alloy composition (defined in this context as the percentage of the dissolving component) necessary for a structure to fully dealloy; this does not necessarily imply porosity evolution and only asks the question whether or not the dissolving liquid can travel from one end of the sample to the other. Another way of considering this problem is whether there is a network/chain of atoms [of the dissolving component], which goes through the entire sample. If the composition is below the parting limit the structure will not fully dealloy, and if the composition is above the parting limiting it will fully dealloy.

For electrochemical dealloying of $\mathrm{Ag}_{\mathrm{x}} \mathrm{Au}_{1-\mathrm{x}}$ the observed parting limit is $\sim 55$ at.\% $\mathrm{Ag}$, however, the theoretical site percolation threshold for an fcc lattice is $\sim 20$ at. $\%$. Recent work by Artymowicz et al. has proposed to explain this difference by considering high-density clusters, which suggests there is a geometric criterion that a percolation chain of Ag atoms needs to be a certain width in order for bulk dealloying to occur [24,26]. Kinetic factors such as surface diffusion 
and dissolution can also alter the observed threshold. In particular, one would expect the smoothing effect of surface diffusion to cause the parting limit to be larger than the theoretical site percolation threshold. However, this effect has not been studied quantitatively to date. For the case of the bcc lattice, $p_{c} \approx 24.5$ at. $\%$, and we may expect the parting limit to be higher in bcc compared to fcc dealloying systems.

In this study we did not characterize the parting limit in detail; however, our data suggests it likely sits around 40 at.\% $\mathrm{Ti}$. We attempted to dealloy $\mathrm{Ti}_{30} \mathrm{Ta}_{70}$ and $\mathrm{Ti}_{20} \mathrm{Ta}_{80}$ samples but did not observe any significant dissolution. Samples from both alloys were immersed into molten $\mathrm{Cu}$ for several minutes, and we only observed small channels of $\mathrm{Cu}$ near the surface of the sample which were no wider than $500 \mathrm{~nm}$ and no longer than tens of microns; this agrees with the dendriticvoid morphology observed in ref [17] for compositions below the parting limit. Previous work in electrochemical dealloying has demonstrated that surface dealloying will always occur due to low coordination at the surface, but this does not imply that the sample will fully dealloy. Additionally, grain boundaries are poorly coordinated and are likely dissolved at compositions below the parting limit - analogous to grain boundary embrittlement in pure elemental systems. We were able to achieve bulk dealloying of $\mathrm{Ti}_{40} \mathrm{Ta}_{60}$ alloys, however the measured activation barrier for this composition was almost twice that of the $\mathrm{Ti}_{55} \mathrm{Ta}_{45}$ and $\mathrm{Ti}_{70} \mathrm{Ta}_{30}$ alloys; for this reason we speculate 40 at.\% Ti is just above the parting limit.

This parting limit is surprising because it is lower than most electrochemical dealloying systems, however there are special cases such as $\mathrm{Al}-\mathrm{Cu}$ and $\mathrm{Zn}-\mathrm{Cu}$ which have thresholds around 20 at.\% [27]. Our system could be a special case like Al/Zn-Cu, however a much older LMD study observed a similar percolation threshold, $30 \mathrm{at} . \%<p_{c}<40 \mathrm{at} . \%$, in a familiar electrochemical dealloying system, $\mathrm{Au}-\mathrm{Cu}$, which has an electrochemical parting limit of 55 at.\% [13]. We are 
cautious to make any definite conclusions as to the origin of this lower parting limit, and future work is necessary to fully understand the concept of the parting limit in LMD systems.

\subsection{Ligament Size and Morphology}

\subsubsection{Ligament coarsening}

We observed a significant amount of ligament coarsening during LMD in molten $\mathrm{Cu}$, with nmsized features at the interface and $\mu \mathrm{m}$-sized features at the edge of the sample. Error! Reference source not found.a,b contrasts the ligament size in a Ti40 Ta60 sample where the ligaments are $\sim 80$ $\mathrm{nm}$ at the interface and rapidly increase to $\sim 1 \mu \mathrm{m}$ at the edge of the sample. The morphology of the structures, see Section 3.6.2, was composition dependent, but in general the lengthscale was approximately the same as it varied away from the interface regardless of the composition.

It was shown in previous work that coarsening in nanoporous metals consists of two concurrent surface diffusion-mediated processes: surface smoothening and ligament pinch-off events [21,28].

In an ideal case both have a $t^{1 / 4}$ dependence, but the mutual dependence of these two processes leads to a more complicated relationship for the surface evolution in nanoporous metals. While fitting coarsening in porous metals with a simple surface-diffusion-dominated scaling law is an oversimplification, it enables us to measure an activation energy associated with coarsening and use it here to comment on ligament coarsening during liquid metal dealloying.

Error! Reference source not found.c shows the ligament size versus dealloyed depth for $\mathrm{Ti}_{40} \mathrm{Ta}_{60}$, dealloyed for 20 seconds at $1160,1240,1305$, and $1360{ }^{\circ} \mathrm{C}$. Using Eq. (9) and our values listed in Table 1 for 20 seconds we can map the ligament size, $\lambda$, to a function of time, included as Error! Reference source not found.d. We assumed a general power-law relationship for coarsening: 


$$
\lambda=\left(k t D_{s}\right)^{n}=\left(\frac{k D_{S} x_{i}^{2}}{4 p D_{L}}\right)^{n}
$$

where $k$ is a fitting constant and $D_{s}$ is the surface diffusivity, which has a general Arrhenius form $D_{s}=D_{s}^{0} \exp \left(-E_{A}^{S} / k_{B} T\right)$. We only show the data for one composition, however the associated activation energies were reasonably close: $\operatorname{Ti}_{70} \operatorname{Ta}_{30}\left(E_{a}=0.79 \mathrm{eV}\right), \operatorname{Ti}_{55} \operatorname{Ta}_{45}\left(E_{a}=0.75 \mathrm{eV}\right)$, and $\operatorname{Ti}_{40} \operatorname{Ta}_{60}\left(E_{a}=1.01 \mathrm{eV}\right)$. These activation energies are similar, but slightly higher, to the activation energies we calculated for Ti diffusion in liquid $\mathrm{Cu}$, but it should be emphasized that the powerlaw exponent $n$ for surface diffusion is lower than diffusion in the liquid phase.

It should be apparent from Error! Reference source not found.c,d that there are two distinct ligament coarsening regions. There appears to be a slight lag in the initial ligament size as a function of dealloying depth in Region I (Error! Reference source not found.c) however this disappears when we convert to an estimated time (Error! Reference source not found.d). It was shown in simulations that the ligament size will always lag a reduction in genus, but this effect is difficult to conclude without estimating an average genus per volume [21]. In Region I (Error! Reference source not found.d) we observe a power-law exponent close to the ideal for coarsening ( $n \approx 0.29$ ), which is reasonable because at longer times coarsening should be limited by mass transport across the surface and not genus reduction events. We attribute the higher exponent to the fact that the ligament surface is undergoing an additional roughening event from the dissolution of Ti. This is similar to the case of surface evolution/coarsening when the solid is in equilibrium with the vapor phase $(n \approx 0.33)$ [29]. In Region II (Error! Reference source not found.c,d), however, we barely notice any increase in the ligament size for a given temperature, which we attribute to the structure approaching a genus of zero (over the scale of measurement). The 
ligaments at the edge of the samples have very low genus/volume values and can no longer increase their size through surface diffusion-mediated events. It may be expected for coarsening in Region II to occur via Ostwald ripening $\left(\langle\lambda\rangle^{3}-\left\langle\lambda_{0}\right\rangle^{3} \propto t\right)$, but there is not enough data in Region II to get a meaningful result. This behavior is expected because the Ostwald ripening relationship requires [some] solubility of the particle in the liquid phase, however Ta is immiscible in molten $\mathrm{Cu}$. As a result the only way the average ligament size can increase is by two ligaments coming into contact and fusing together. This appears to be a very slow process, and the ligament size does not increase significantly in Region II.

\subsubsection{Morphology}

It has been noted by several different authors, and is generally accepted, that the bicontinuous structure in nanoporous metals is self-similar, i.e. that the morphology is the same regardless of the lengthscale. In fact, for electrochemical dealloying in the traditional alloy range, $\mathbf{4 0 - 8 5}$ at\% of the dissolving component, there is little compositional effect on the morphology [30]. Dealloying $\mathrm{Ag}_{60} \mathrm{Au}_{40}$ or $\mathrm{Ag}_{75} \mathrm{Au}_{35}$ yields the same porous $\mathrm{Au}$ structure, with minor differences in ligament composition and the ligament:pore ratio. This is not the case for liquid metal dealloying and dramatically different structures have been observed in Ti-Ta alloys dealloyed in $\mathrm{Cu}$ and are discussed in detail in ref [12]. Briefly, ref [12] observed a morphology transition from Ta-rich droplets to a connected, porous Ta-rich network as the parent alloy composition changed from $\mathrm{Ti}_{95} \mathrm{Ta}_{5}$ to $\mathrm{Ti}_{65} \mathrm{Ta}_{35}$. These morphological differences could be due to the high homologous temperature during dealloying, but that remains unclear. In this current study we are only examining connected structures, and report subtle changes in the morphology. Specifically we see an increase in the 2D connectivity of the structures as we increase the Ta content of the parent 
alloy, which in turn affected the stability of the Ta network at longer times, Error! Reference source not found. We are only examining the $(x, y)$ plane, however we expect the microstructure to be isotropic and the degree of connectivity on $(y, z)$ plane should be similar to the one we observe. It should also be noted that the since dealloying is a layer-by-layer process the $(x, y)$ plane is more relevant for assessing the mechanical integrity of the structure.

We observed good mechanical integrity when the Ta concentration was greater than $\sim 30$ at.\%, owing to the connectivity in the porous network, which is a major reason why this work focuses on Ta-rich alloys. Samples with lower Ta concentrations were mechanically stable for short immersion times (less than 10 seconds), but fell apart during longer immersions (greater than 20 seconds). Only samples with at least 30 at.\% Ta were robust enough to hold together during long immersion experiments (up to 120 seconds). Ligament pinch-off mediated coarsening decreases the connectivity of the porous structure with increasing time, and eventually ruins the mechanical integrity of the sample. Not only did the connectivity depend on time but also on the Ta composition. For comparison, Error! Reference source not found. shows the connectivity of the ligaments change at the edge of the sample from disconnected blobs in the $\mathrm{Ti}_{70} \mathrm{Ta}_{30}$ sample to a highly connected structure in the $\mathrm{Ti}_{40} \mathrm{Ta}_{60}$ sample. This effect is quite significant in $\mathrm{Cu}$ baths, however it should be less obvious in lower-melting point baths where the ligaments do not coarsen significantly past their formation size.

It was mentioned in Section 3.4 that the activation energy for the dealloying process of the $\mathrm{Ti}_{40} \mathrm{Ta}_{60}$ was quite high, almost twice that for $\mathrm{Ti}_{55} \mathrm{Ta}_{45}$ and $\mathrm{Ti}_{70} \mathrm{Ta}_{30}$. It is possible that this composition indicates the rate-limiting process transitions from Ti diffusion in the melt to surface diffusion/reorganization at the dealloying front. The activation energy calculated for the dealloying 
depth kinetics was very similar to the activation energy for coarsening in Section 3.6.1., but it is very difficult to deconvolute pinch-off events from surface smoothening events

\subsubsection{Homologous Temperature Effects}

It has been demonstrated that the ligament size increases with increasing time and temperature, but we have not discussed methods to refine the ligament size. The goal of this section is to show that if we lower the temperature during dealloying we can suppress surface diffusion and maintain nm-sized ligaments. In principle, this should not affect the dissolution rate significantly because the activation energy for Ti diffusion away from the interface is small. By adding $\mathrm{Ag}$ and $\mathrm{Bi}$ to molten $\mathrm{Cu}$ we can fabricate molten baths with lower melting points across the range $400-1000{ }^{\circ} \mathrm{C}$; we specify adding components to the bath because $\mathrm{Cu}, \mathrm{Ag}$, and $\mathrm{Bi}$ phase separate in the solid phase. Due to the difficulty in imaging nanocomposites we dissolved out the liquid metal phase, and Error! Reference source not found. shows nanoporous Ta generated from dealloying in a $\mathrm{Cu}_{32} \mathrm{Ag}_{68}$ bath (Error! Reference source not found.a), and $\mathrm{Cu}_{20} \mathrm{Ag}_{40} \mathrm{Bi}_{40}$ bath (Error! Reference source not found.b). Each sample was dealloyed for a significant time, $~ 60$ minutes, to demonstrate that the feature size we observe is an upper limit for the dealloying temperature, but the dealloyed depth was only $\sim 150 \mu \mathrm{m}$.

Although the dealloying rate will not decrease substantially at a lower temperature, we were unable to dealloy to large depths because it was difficult to design an alloy bath that also maintained a high solubility of the dissolving component. Across this temperature range, $\mathrm{Bi}(\ell)$ and $\mathrm{Ag}(\ell)$ have at most 4 and 2 at.\% Ti solubility, respectively, although it is unclear how $\mathrm{Cu}$ affects the solubility of this mixture. As a result, the dealloying rate becomes very sensitive to the size of the bath, and large quantities are needed to dealloy thin samples. Future work will need to 
explore balancing the addition of components to the liquid bath to suppress coarsening without dramatically reducing the dealloying rate.

\section{Summary and Conclusions}

We examined the kinetics of liquid metal dealloying by varying the parent alloy composition, dealloying temperature, and dealloying time. The values measured for the diffusivity and activation energy confirm our initial hypothesis that the rate-limiting step in the dealloying process is Ti diffusion away from the dealloying interface and out of the dealloyed structure. It is possible, however, that a second mechanism may become dominant when the alloy composition is close to the parting limit. Differences between our calculated diffusivites and activation energies and the literature value for Ti diffusion in liquid $\mathrm{Cu}$ can be attributed to ligament coarsening adding extra Ti to the $\mathrm{Cu}$ bath. Ligament coarsening is very fast at this temperature, increasing from $80 \mathrm{~nm}$ to $1 \mu \mathrm{m}$ in just 10 seconds. To stymie coarsening we performed LMD experiments in lower-melting point baths, however the low Ti solubility in these baths made it difficult to dealloy to large depths (of order $1 \mathrm{~mm}$ ). In summary, the present study has developed a quantitative framework for predicting the kinetics of dissolution and morphological evolution during liquid metal dealloying, which we have shown to be coupled processes. The results lay the groundwork for future research in this field, which still needs to overcome the challenge of fabricating bulk structures with nmsized ligaments.

\section{Acknowledgement}

I.M. would like to thank J.M. for helpful discussions, feedback, love and support. I.M., B.G., and J.E. are grateful for funding from the National Science Foundation under grant DMR-1402726 
and DMR-1003901. P.-A.G. and A.K. acknowledge the support of grant DE-FG02-07ER46400 from the U.S. Department of Energy, Office of Basic Energy Sciences. 


\section{Appendix A.}

\section{A.1 Initial Dissolution Problem}

First, we introduce the general case of dissolution of an alloy into a liquid bath: a binary alloy, Ti-Ta (with Ti concentration $c_{s}^{T i}$ ) on the left of the system is dissolved in a liquid bath, Ti-Cu (with Ti concentration $c_{B}^{T i}$ ) on the right of the system (see Error! Reference source not found.). The position of the solid/liquid interface is noted $x_{i}(t)$ and we take $x_{i}(t=0)=0$. We assume that the diffusion is frozen in the solid and the dissolution front remains planar, moving towards negative $x$ at a rate $v_{i}$. We note $c(x)$, the Ti concentration in the liquid (i.e. $x>x_{i}$ ), follows a simple diffusion relation:

$$
\partial_{t} c=D_{L} \partial_{x}^{2} c
$$

where $D_{L}$ is the diffusivity of $\mathrm{Ti}$ in liquid $\mathrm{Cu}$. The interface velocity $v_{i}$ is controlled by the concentration flux at the solid/liquid interface:

$$
v_{i}\left(c_{S}^{T i}-c_{L}^{T i}\right)=\left.D_{L} \frac{\partial c}{\partial x}\right|_{i},
$$

where $c_{L}^{T i}$ is the Ti concentration in the liquid phase at the interface. This interface concentration is assumed to remain constant in time, consistent with the physical picture that dissolution is slow enough for the solid-liquid interface to be in quasi-local chemical equilibrium [11]. The Ti concentration at the interface satisfies one of the boundary conditions for the concentration field $c(x)$, but there are several possible boundary conditions for the $\mathrm{Cu}$ bath. The most simple and natural boundary condition is to consider an infinite bath where Ti can diffuse into, Eq. (A3). Although Eq. (A3) is straightforward, the actual dealloying experiments use RF induction to heat the liquid bath, which induces convection currents, Eq. (A4). Outside of the dealloyed region, i.e. 
$x>0$, it is reasonable to conclude the convection currents lead to complete mixing, but the length scale of the ligaments protect the dealloyed region from these convection currents and the $\mathrm{Ti}$ concentration of the liquid in the dealloyed region follows purely diffusive dynamics. As a result, we have two different boundary conditions for the Cu bath depending on whether or not we account for convection currents:

$$
\begin{aligned}
& c(x=+\infty, t)=c_{B}^{T i}, \\
& c(x \geq 0, t)=c_{B}^{T i} .
\end{aligned}
$$

We introduce the reduced concentration field defined as:

$$
u(x, t)=\frac{c(x, t)-c_{L}^{T i}}{c_{S}^{T i}-c_{L}^{T i}}
$$

Eqs. (A3) and (A4) and the boundary conditions can be rewritten with $u$ leading to the following set of equations:

$$
\begin{aligned}
& \partial_{t} u=D_{L} \partial_{x}^{2} u \\
& v_{i}=\left.D_{L} \frac{\partial u}{\partial x}\right|_{i} \\
& u\left(x=x_{i}\right)=0 \\
& u(x=+\infty)=-\Omega \\
& \text { or, } \\
& u(x=0)=-\Omega,
\end{aligned}
$$

where $\Omega=\frac{c_{L}^{T i}-c_{B}^{T i}}{c_{S}^{T i}-c_{L}^{T i}}$ is the analog of the supersaturation in solidification problems. 
We introduce the dimensionless coordinate $z(x, t)=x / x_{i}(t)$ and assume that $u(x, t)$ can be written as a function of $z$, i.e. $u(x, t)=f(z)$. Eqs. (A6)-(A10) can be written with $f$ by introducing the dimensionless Péclet number of the problem $p=x_{i} v_{i} / 2 D_{L}$ :

$$
\begin{aligned}
& 2 p z \frac{d f}{d z}+\frac{d^{2} f}{d z^{2}}=0 \\
& 2 p=\left.\frac{\partial f}{\partial z}\right|_{i} \\
& f(z=1)=0
\end{aligned}
$$

and

$$
f(z=-\infty)=-\Omega
$$

or,

$$
f(z=0)=-\Omega
$$

We define $g=d f / d z$ and Eq. (A11) admits solutions of the form $g(z)=A e^{-p z^{2}}$ with A as a constant that can be determined from Eq. (A12). We finally obtain a solution of the form:

$$
g(z)=2 p e^{p} e^{-p z^{2}}
$$

\section{A.2 Dissolution Kinetics: Diffusion in an Infinite Bath}

Using boundary conditions Eqs. (A13) and (A14), we can integrate $g(z)$ between $-\infty$ and 1 , obtaining:

$$
\int_{-\infty}^{1} g(z) d z=f(1)-f(-\infty)
$$




$$
2 p e^{p} \int_{-\infty}^{1} e^{-p z^{2}} d z=\Omega
$$

After introducing the change of variable $\xi=z \sqrt{p}$ and using the fact that $\int_{-\infty}^{0} e^{-\xi^{2}} d \xi=\sqrt{\pi} / 2$, we finally obtain the following relation between the Péclet number $p$ and $\Omega$ :

$$
\Omega=\sqrt{\pi p} e^{p}(1+\operatorname{erf}(\sqrt{p}))=\Gamma_{1}(p)
$$

where $\operatorname{erf}(x)=\frac{2}{\sqrt{\pi}} \int_{0}^{x} e^{-u^{2}} d u$ is the Gauss error function. Because the function $\Gamma_{1}(p)$ is bijective for positive reals, a single constant Péclet number $p=\Gamma_{1}^{-1}(\Omega)$ corresponds to a fixed supersaturation. From the expression of the Péclet number, $p=x_{i} v_{i} / 2 D_{L}$, we can integrate in time and deduce the evolution of the interface position $x_{i}(t)$ :

$$
x_{i}(t)=-\sqrt{4 D p t} .
$$

The evolution of the solute field in the liquid $u(x, t)$ can also be deduced by integrating Eq. (A16) between $-\infty$ and an arbitrary $z$ :

$$
f(z)=-\Omega+\sqrt{\pi p} e^{p}(1+\operatorname{erf}(z \sqrt{p})) .
$$

Using the fact that $f(z)=u(x, t)$ and the definition of $z=x / x_{i}(t)=-x / \sqrt{4 D p t}$, we obtain the following relation:

$$
u(x, t)=\sqrt{p \pi} e^{p}\left(\operatorname{erf}\left(-\frac{x}{\sqrt{4 D t}}\right)-\operatorname{erf}(\sqrt{p})\right)
$$

or in terms of $c(x, t)$ :

$$
c(x, t)=c_{L}^{T i}+\left(c_{S}^{T i}-c_{L}^{T i}\right) \sqrt{p \pi} e^{p}\left(\operatorname{erf}\left(-\frac{x}{\sqrt{4 D t}}\right)-\operatorname{erf}(\sqrt{p})\right) .
$$




\section{A.3 Dissolution Kinetics: Accounting for Convection}

Using boundary conditions Eqs. (A13) and (A15), we can integrate $g(z)$ between 0 and 1 , obtaining:

$$
\begin{aligned}
& \int_{-\infty}^{1} g(z) d z=f(1)-f(0), \\
& 2 p e^{p} \int_{0}^{1} e^{-p z^{2}} d z=\Omega .
\end{aligned}
$$

We obtain the following relation between the Péclet number $p$ and $\Omega$ :

$$
\Omega=\sqrt{\pi p} e^{p} \operatorname{erf}(\sqrt{p})=\Gamma_{2}(p) .
$$

Once again, a single constant Péclet number $p=\Gamma_{2}^{-1}(\Omega)$ corresponds to a fixed supersaturation. From the expression of the Péclet number, $p=x_{i} v_{i} / 2 D_{L}$, we can integrate in time and deduce the evolution of the interface position $x_{i}(t)$ :

$$
x_{i}(t)=-\sqrt{4 D p t}
$$

The evolution of the solute field in the liquid $u(x, t)$ can also be deduced by integrating Eq. (A16) between $-\infty$ and an arbitrary $z$ :

$$
f(z)=-\Omega+\sqrt{\pi p} e^{p} \operatorname{erf}(z \sqrt{p}) .
$$

Using the fact that $f(z)=u(x, t)$ and the definition of $z=x / x_{i}(t)=-x / \sqrt{4 D p t}$, we obtain the following relation:

$$
u(x, t)=\sqrt{p \pi} e^{p}\left(\operatorname{erf}\left(-\frac{x}{\sqrt{4 D t}}\right)-\operatorname{erf}(\sqrt{p})\right)
$$


or in terms of $c(x, t)$ :

$$
c(x, t)=c_{L}^{T i}+\left(c_{S}^{T i}-c_{L}^{T i}\right) \sqrt{p \pi} e^{p}\left(\operatorname{erf}\left(-\frac{x}{\sqrt{4 D t}}\right)-\operatorname{erf}(\sqrt{p})\right) .
$$

This relation may appear identical to Eq. (A23) however the Péclet number is defined with Eq. (A26) instead of Eq. (A19). 


\section{References}

[1] T. Wada, K. Yubuta, A. Inoue, H. Kato, Dealloying by metallic melt, Mater. Lett. 65 (2011) 1076-1078. doi:10.1016/j.matlet.2011.01.054.

[2] T. Wada, T. Ichitsubo, K. Yubuta, H. Segawa, H. Yoshida, H. Kato, Bulk-nanoporoussilicon negative electrode with extremely high cyclability for lithium-ion batteries prepared using a top-down process, Nano Lett. 14 (2014) 4505-4510.

doi:10.1021/nl501500g.

[3] J.W. Kim, M. Tsuda, T. Wada, K. Yubuta, S.G. Kim, H. Kato, Optimizing niobium dealloying with metallic melt to fabricate porous structure for electrolytic capacitors, Acta Mater. 84 (2015) 497-505. doi:10.1016/j.actamat.2014.11.002.

[4] J.W. Kim, T. Wada, S.G. Kim, H. Kato, Sub-micron porous niobium solid electrolytic capacitor prepared by dealloying in a metallic melt, Mater. Lett. 116 (2014) 223-226. doi:10.1016/j.matlet.2013.11.036.

[5] I. McCue, S. Ryan, K. Hemker, X. Xu, N. Li, M. Chen, et al., Size Effects in the Mechanical Properties of Bulk Bicontinuous Ta/Cu Nanocomposites Made by Liquid Metal Dealloying, Adv. Eng. Mater. (2015) n/a-n/a. doi:10.1002/adem.201500219.

[6] J. Erlebacher, R. Seshadri, Hard Materials with Tunable Porosity, MRS Bull. 34 (2009) 561-568. doi:10.1557/mrs2009.155.

[7] J. Erlebacher, M.J. Aziz, a Karma, N. Dimitrov, K. Sieradzki, Evolution of nanoporosity in dealloying., Nature. 410 (2001) 450-453. doi:10.1038/35068529.

[8] J. Erlebacher, An Atomistic Description of Dealloying, J. Electrochem. Soc. 151 (2004) C614. doi:10.1149/1.1784820.

[9] I. McCue, E. Benn, B. Gaskey, J. Erlebacher, Dealloying and Dealloyed Materials, Annu. Rev. Mater. Res. 46 (2016) annurev-matsci-070115-031739. doi:10.1146/annurevmatsci-070115-031739.

[10] T. Wada, H. Kato, Three-dimensional open-cell macroporous iron, chromium and ferritic stainless steel, Scr. Mater. 68 (2013) 723-726. doi:10.1016/j.scriptamat.2013.01.011.

[11] M. Tsuda, T. Wada, H. Kato, Kinetics of formation and coarsening of nanoporous $\alpha-$ titanium dealloyed with Mg melt, J. Appl. Phys. 114 (2013) 113503. doi:10.1063/1.4821066.

[12] P.-A. Geslin, I. McCue, B. Gaskey, J. Erlebacher, A. Karma, Topology-generating Interfacial Pattern Formation During Liquid Metal Dealloying, 2015.

[13] J.. Harrison, C. Wagner, The attack of solid alloys by liquid metals and salt melts, Acta Metall. 7 (1959) 722-735. doi:10.1016/0001-6160(59)90178-6.

[14] B. Šarler, Stefan's work on solid-liquid phase changes, Eng. Anal. Bound. Elem. 16 (1995) 83-92. doi:10.1016/0955-7997(95)00047-X. 
[15] Y.C.K. Chen-Wiegart, S. Wang, I. McNulty, D.C. Dunand, Effect of Ag-Au composition and acid concentration on dealloying front velocity and cracking during nanoporous gold formation, Acta Mater. 61 (2013) 5561-5570. doi:10.1016/j.actamat.2013.05.039.

[16] Y.C.K. Chen-Wiegart, S. Wang, W.K. Lee, I. McNulty, P.W. Voorhees, D.C. Dunand, In situ imaging of dealloying during nanoporous gold formation by transmission X-ray microscopy, Acta Mater. 61 (2013) 1118-1125. doi:10.1016/j.actamat.2012.10.017.

[17] Q. Chen, K. Sieradzki, Mechanisms and Morphology Evolution in Dealloying, J. Electrochem. Soc. 160 (2013) C226-C231. doi:10.1149/2.064306jes.

[18] J.C. Dibbern, Interdiffusivity in Titanium-Tantalum Alloys Processed at 1473 K, Massachusetts Institute of Technology, 2007. http://hdl.handle.net/1721.1/44821.

[19] D.B. Butrymowicz, J.R. Manning, M.E. Read, Diffusion in copper and copper alloys. Part III. Diffusion in systems involving elements of the groups IA, IIA, IIIB, IVB, VB, VIB, and VIIB, J. Phys. Chem. Ref. Data. 4 (1975) 177. doi:10.1063/1.555516.

[20] X.-L. Ye, N. Lu, X.-J. Li, K. Du, J. Tan, H.-J. Jin, Primary and Secondary Dealloying of $\mathrm{Au}(\mathrm{Pt})-\mathrm{Ag}$ : Structural and Compositional Evolutions, and Volume Shrinkage, J. Electrochem. Soc. 161 (2014) C517-C526. doi:10.1149/2.0131412jes.

[21] J. Erlebacher, Mechanism of coarsening and bubble formation in high-genus nanoporous metals, Phys. Rev. Lett. 106 (2011) 1-4. doi:10.1103/PhysRevLett.106.225504.

[22] L.H. Qian, M.W. Chen, Ultrafine nanoporous gold by low-temperature dealloying and kinetics of nanopore formation, Appl. Phys. Lett. 91 (2007) 083105. doi:10.1063/1.2773757.

[23] K. Sieradzki, The fracture strength of solids near the percolation threshold, J. Phys. C Solid State Phys. 18 (1985) L855-L856. doi:10.1088/0022-3719/18/27/002.

[24] K. Sieradzki, R.R. Corderman, K. Shukla, R.C. Newman, Computer simulations of corrosion: Selective dissolution of binary alloys, Philos. Mag. A. 59 (1989) 713-746. doi:10.1080/01418618908209817.

[25] J. Rugolo, J. Erlebacher, K. Sieradzki, Length scales in alloy dissolution and measurement of absolute interfacial free energy., Nat. Mater. 5 (2006) 946-949. doi:10.1038/nmat1780.

[26] D.M. Artymowicz, J. Erlebacher, R.C. Newman, Relationship between the parting limit for de-alloying and a particular geometric high-density site percolation threshold, Philos. Mag. 89 (2009) 1663-1693. doi:10.1080/14786430903025708.

[27] K. Sieradzki, The Relationship Between Dealloying and Transgranular Stress-Corrosion Cracking of $\mathrm{Cu}-\mathrm{Zn}$ and Cu-Al Alloys, J. Electrochem. Soc. 134 (1987) 1635. doi:10.1149/1.2100726.

[28] K. Kolluri, M.J. Demkowicz, Coarsening by network restructuring in model nanoporous gold, Acta Mater. 59 (2011) 7645-7653. doi:10.1016/j.actamat.2011.08.037.

[29] W.W. Mullins, Theory of Thermal Grooving, J. Appl. Phys. 28 (1957) 333. doi:10.1063/1.1722742. 
[30] J. Snyder, J. Erlebacher, Kinetics of Crystal Etching Limited by Terrace Dissolution, J. Electrochem. Soc. 157 (2010) C125. doi:10.1149/1.3280299. 


\section{Figures and Table}

Fig. 1. Scanning electron microscopy (SEM) micrograph of Ti55Ta45 samples dealloyed for 20 seconds at four temperatures.

Fig. 2. Dealloying depth versus time for the samples in this study.

Fig. 3. Comparison of $\mathrm{Ti}$ concentration in the $\mathrm{Cu}$ phase versus distance away from the dealloying interface between different $\mathrm{Ti}_{\mathrm{x}} \mathrm{Ta}_{1-\mathrm{x}}$ alloy compositions.

Fig. 4. Illustration of liquid metal dealloying.

Fig. 5. Comparison of Ti concentration in the $\mathrm{Cu}$ phase versus distance away from the dealloying interface.

Fig. 6. Ti Concentration versus distance away from the dealloying interface for $\operatorname{Ti}_{70} \mathrm{Ta}_{30}$ at 1240 ${ }^{\circ} \mathrm{C}$.

Fig. 7. Collapsed dealloying depth versus scaled time.

Fig. 8. Effects of grain boundaries on dealloying.

Fig. 9. Ligament coarsening during LMD using $\mathrm{Ti}_{40} \mathrm{Ta}_{60}$ as an example.

Fig. 10. Difference in morphology at the edge of the sample.

Fig. 11. Ligament size temperature dependence.

Table 1. Kinetic parameters determined in this study. 

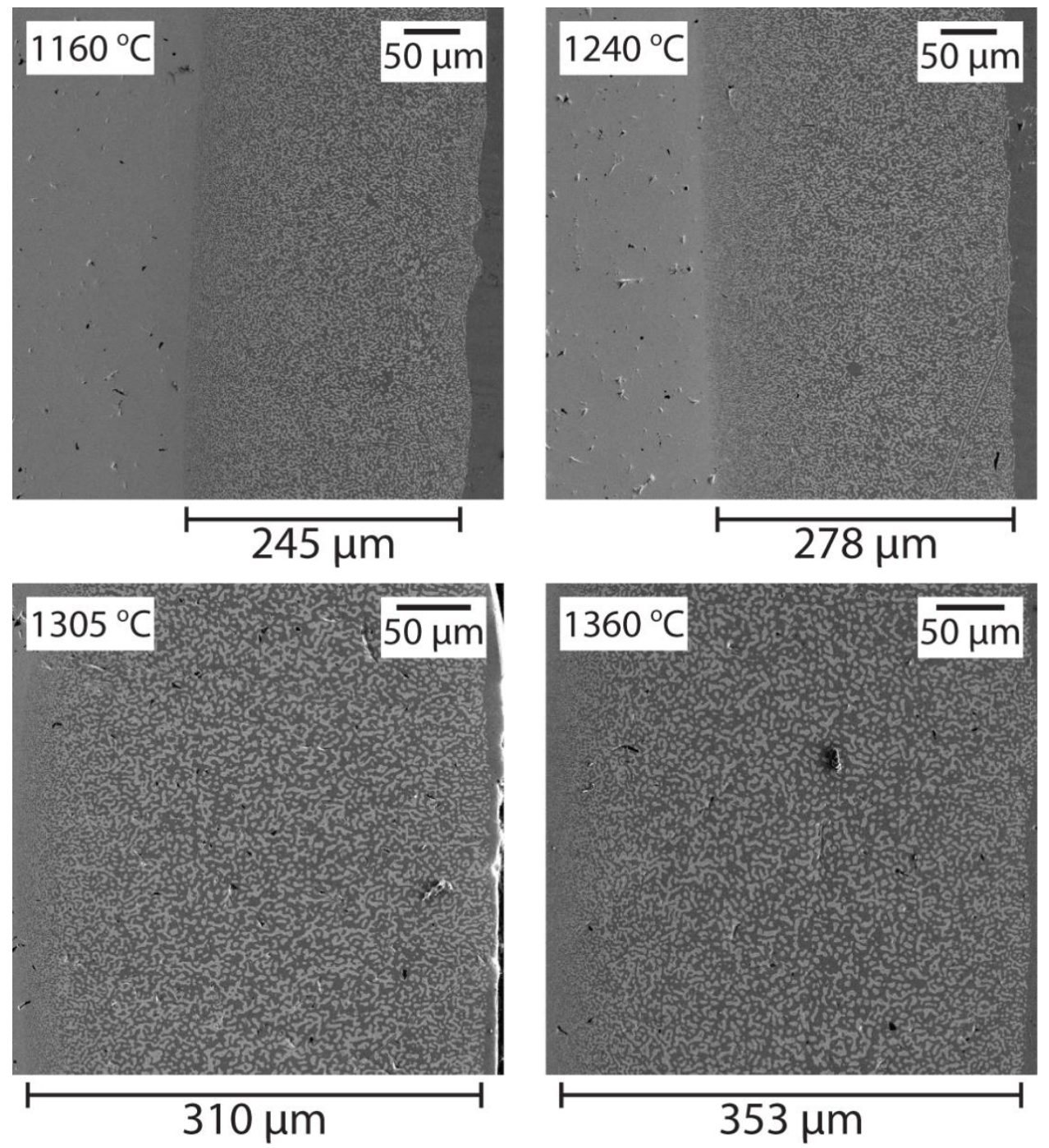

Fig. 1. Scanning electron microscopy (SEM) micrographs of $\mathrm{Ti}_{55} \mathrm{Ta}_{45}$ samples dealloyed in molten $\mathrm{Cu}$ for 20 seconds at four temperatures. The dealloying interface is sharp and flat, and the dealloyed region has a natural contrast due to the different compositions; the dark phase is $\mathrm{Cu}$ and the light phase is Ta. The top two images were taken at 200x magnification and the bottom two were taken at 270x magnification, but it can be seen that dealloying depth increases with increasing time. 

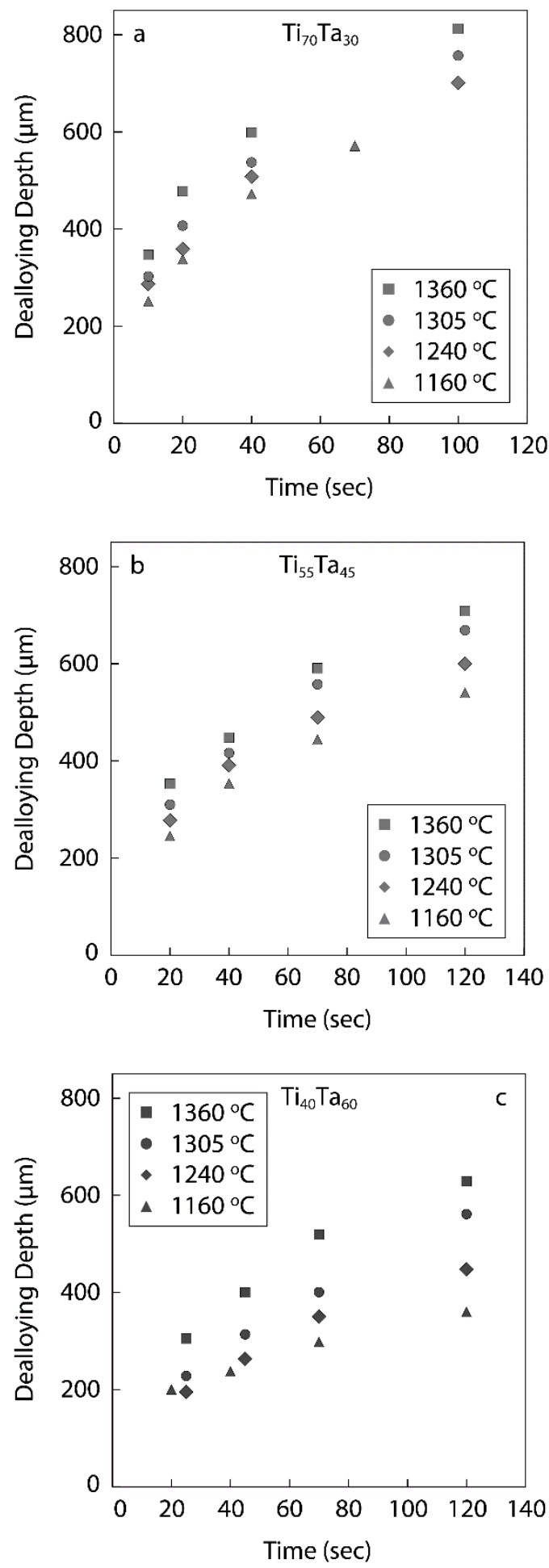

Fig. 2. Dealloying depth versus time for the samples in this study. We studied four temperatures: $1160{ }^{\circ} \mathrm{C}$ (triangle), $1240{ }^{\circ} \mathrm{C}$ (diamond), $1305^{\circ} \mathrm{C}$ (circle), and $1360^{\circ} \mathrm{C}$ (square); four times $(10-120$ seconds); and three compositions: (a) $\mathrm{Ti}_{70} \mathrm{Ta}_{30}$ (blue), (b) $\mathrm{Ti}_{55} \mathrm{Ta}_{45}$ (purple), and (c) $\mathrm{Ti}_{40} \mathrm{Ta}_{60}$ (red). 

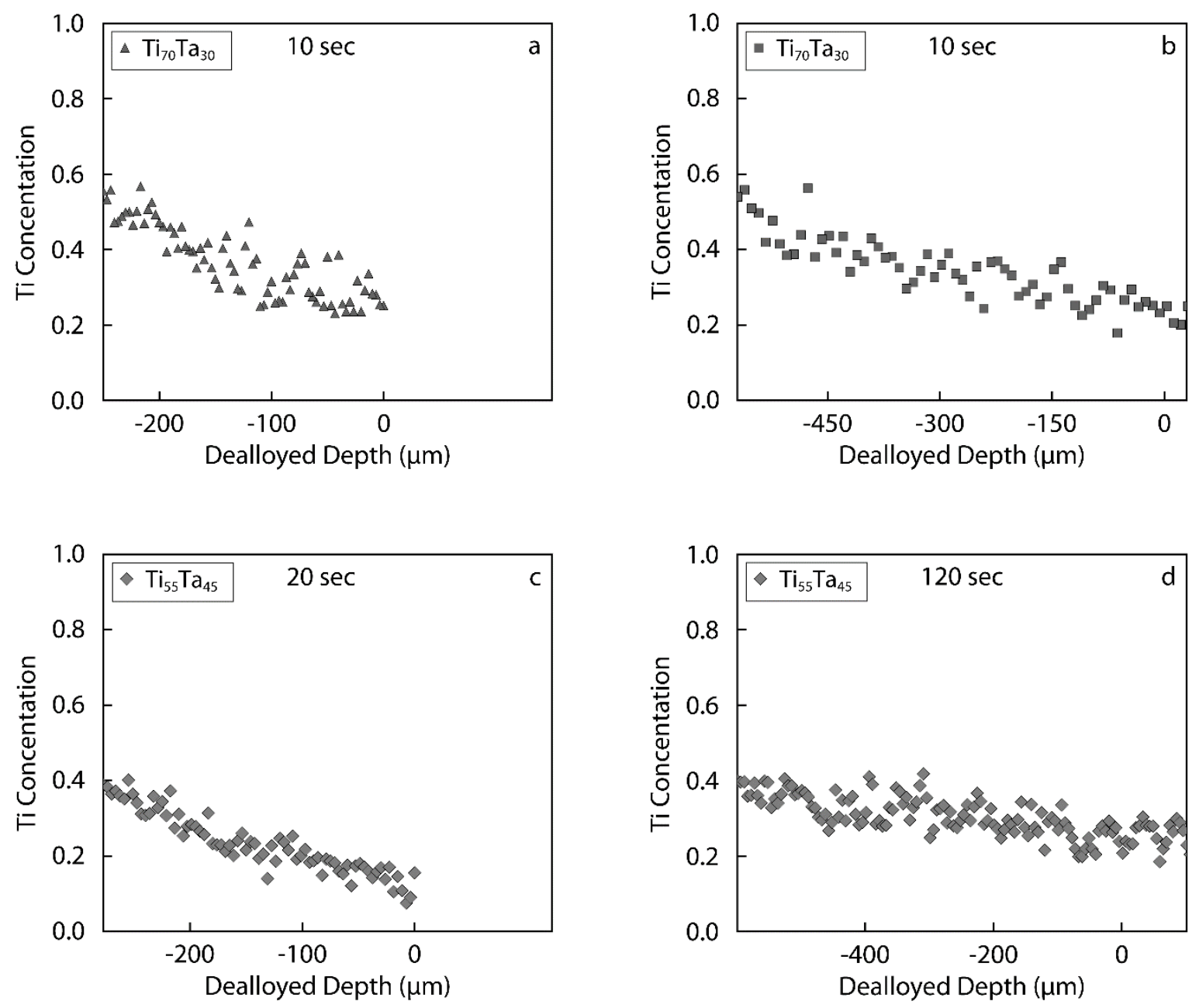

Fig. 3. Comparison of Ti concentration in the $\mathrm{Cu}$ phase versus distance away from the dealloying interface between different $\mathrm{Ti}_{\mathrm{x}} \mathrm{Ta}_{1-\mathrm{x}}$ alloy compositions: (a) $\mathrm{Ti}_{70} \mathrm{Ta}_{30}$ dealloyed for 10 seconds at $1240{ }^{\circ} \mathrm{C}$ (blue triangles); (b) Ti70 Ta30 dealloyed for 10 seconds at $1360{ }^{\circ} \mathrm{C}$ (blue squares); (c) Tis5Ta45 dealloyed for 20 seconds at $1240{ }^{\circ} \mathrm{C}$ (purple diamonds); (d) Ti ${ }_{55} \mathrm{Ta}_{45}$ dealloyed for 120 seconds at $1240{ }^{\circ} \mathrm{C}$ (purple diamonds). To be consistent with the model introduced in the text, $x=0$, represents the edge of the parent alloy, i.e. where it was first dealloyed. The interface of the dealloying front is the left side of each plot. The concentration at the interface is different for each composition but constant across the timescales and temperature range in this study. 


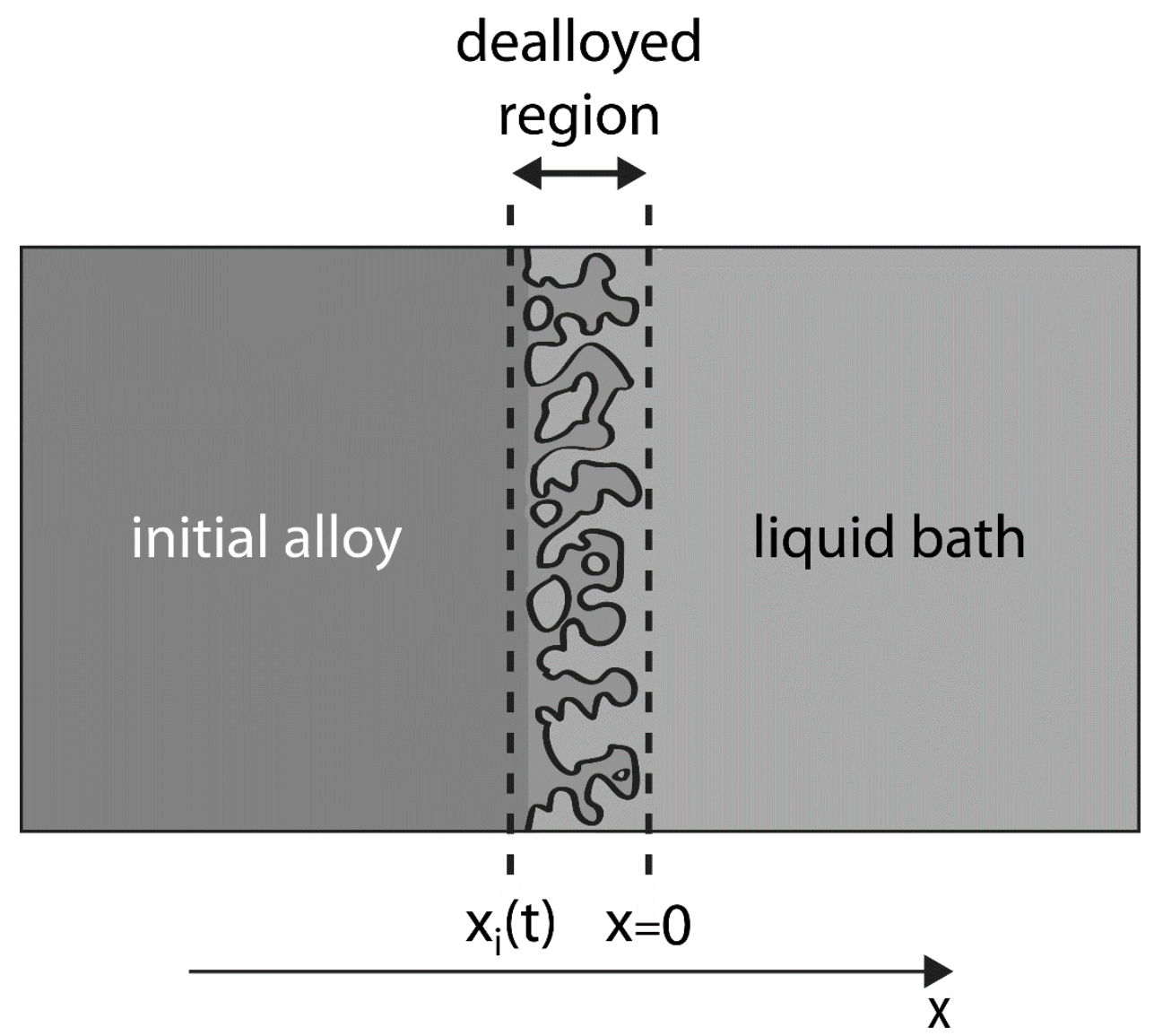

Fig. 4. Illustration of liquid metal dealloying. The dealloyed region (dashed lines) is bounded on the left by molten $\mathrm{Cu}$ (orange) and on the right by undealloyed Ti-Ta (gray). The interface between the solid and the liquid $\mathrm{Cu}$ is initially at $x=0$, and for $t>0$ molten $\mathrm{Cu}$ dissolves Ti out of the solid, moving the interface, $x_{i}(t)$, towards the left $\left(x_{i}(t=\infty)=-\infty\right)$. The Ta atoms are left behind, reorganizing into a porous network. 

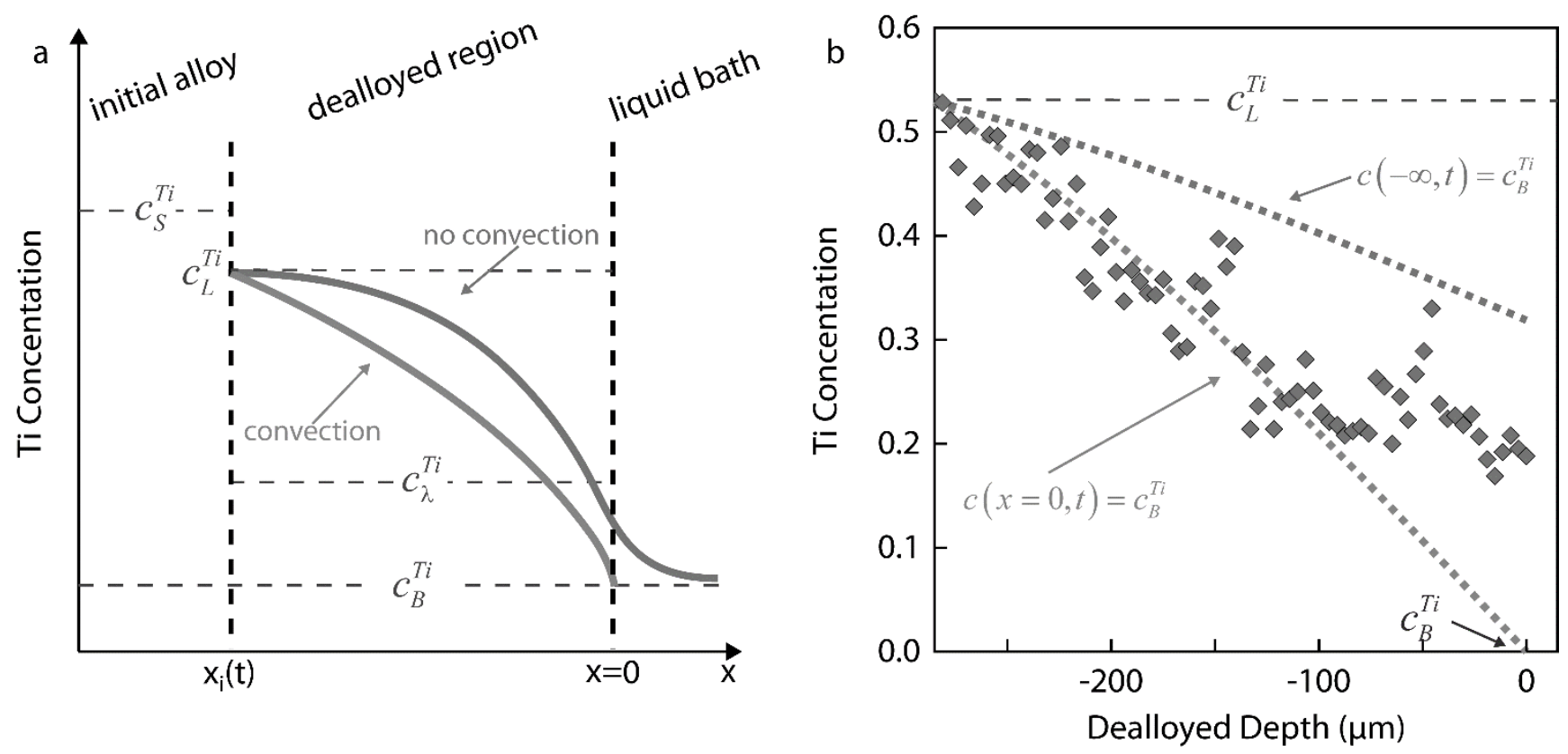

Fig. 5. Comparison of $\mathrm{Ti}$ concentration in the $\mathrm{Cu}$ phase versus distance away from the dealloying interface between the experimental data (blue diamonds) and the analytical model (dashed lines) using the two different boundary conditions. Solution for the Ti concentration profile assuming there is convection in the liquid phase (orange line). Solution for the Ti concentration profile assuming there is no convection in the liquid phase (purple line). (a) Graphic demonstrating the different Ti concentration profiles: $c_{S}^{T i}$ is the concentration in the initial alloy, $c_{L}^{T i}$ is the concentration in the liquid phase, $c_{\lambda}^{T i}$ is the concentration in the Ta-rich ligaments, and $c_{B}^{T i}$ is the concentration in the liquid bath. (b) The analytical model assuming convection matches the experimental data well. The deviation between the model and the experimental data at distances far from the interface is due to ligament coarsening, which adds extra Ti to the $\mathrm{Cu}$ bath. To be consistent with the model introduced in the text, $x=0$, represents the edge of the parent alloy, i.e. where it was first dealloyed. 

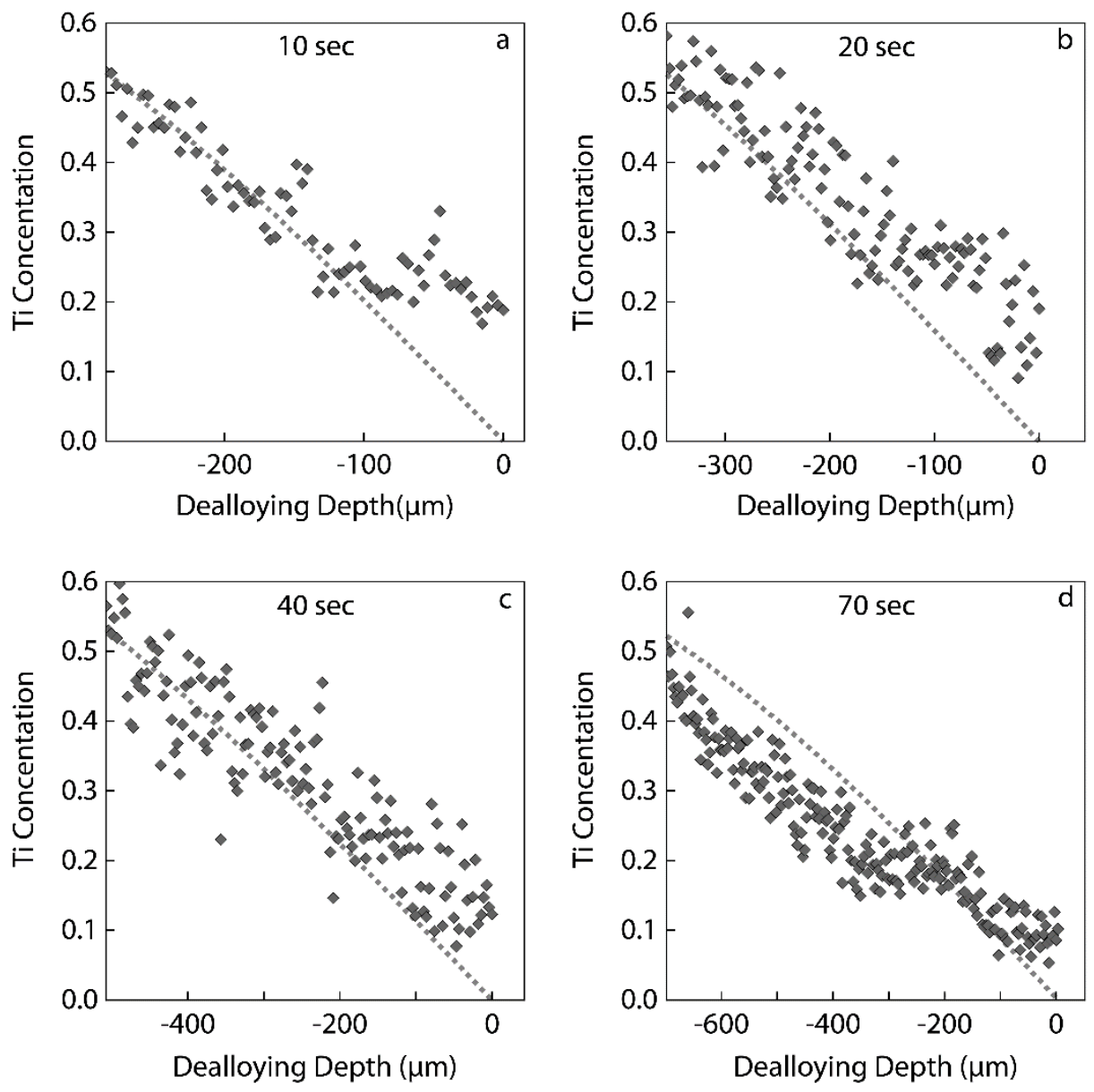

Fig. 6. Ti Concentration versus distance away from the dealloying interface for $\mathrm{Ti}_{70} \mathrm{Ta}_{30}$ at 1240 ${ }^{\circ} \mathrm{C}$. Experimental data (blue diamonds) compared with Eq. Error! Reference source not found. at different times: (a) 10 seconds, (b) 20 seconds, (c) 40 seconds, and (d) 70 seconds. Using an average of the $\mathrm{Ti}$ concentration at the interface, $c_{L}^{T i}=0.5325$, Eq. Error! Reference source not found. was simultaneously fit across all times yielding $D_{L}\left(1240{ }^{\circ} \mathrm{C}\right)=6.5 \times 10^{-5} \mathrm{~cm}^{2} \mathrm{~s}^{-1}$ agreeing well with the literature value $D_{L}^{T i \rightarrow C u}\left(1240{ }^{\circ} \mathrm{C}\right)=7.0 \times 10^{-5} \mathrm{~cm}^{2} \mathrm{~s}^{-1}$. To be consistent with the model introduced in the text, $x=0$ , represents the edge of the parent alloy, i.e. where it was first dealloyed. The interface of the dealloying front is the left side of each plot. 

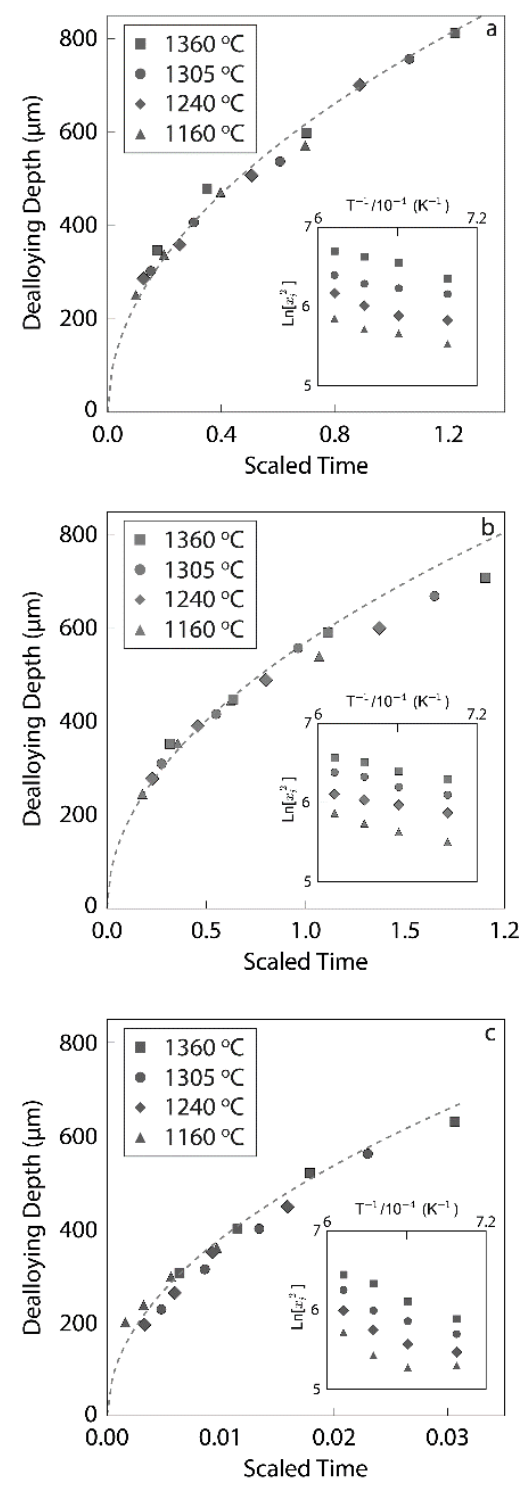

Fig. 7. Collapsed dealloying depth versus scaled time $t^{\prime}=t * \exp \left(-E_{a} / k_{B} T\right)$ for Ti70 Ta30 (blue), $\mathrm{Ti}_{55} \mathrm{Ta}_{45}$ (purple), and $\mathrm{Ti}_{40} \mathrm{Ta}_{60}$ (red). The symbol shape corresponds to the experimental temperature: $1160{ }^{\circ} \mathrm{C}$ (triangle), $1240{ }^{\circ} \mathrm{C}$ (diamond), $1305{ }^{\circ} \mathrm{C}$ (circle), and $1360{ }^{\circ} \mathrm{C}$ (square). Dashed lines: fits to the data using the relationship $x_{i}(t)=\sqrt{4 p D_{L} t}$. The values of $p, D_{0}$, and $E_{a}$ were determined for each data set and are listed in Table 1. (Insets) Arrhenius plots of each dealloying time showing clear linear behavior across our temperature range; deviations from linear behavior are associated with marginally different dealloying times for a given temperature range, see Fig. 2c. 

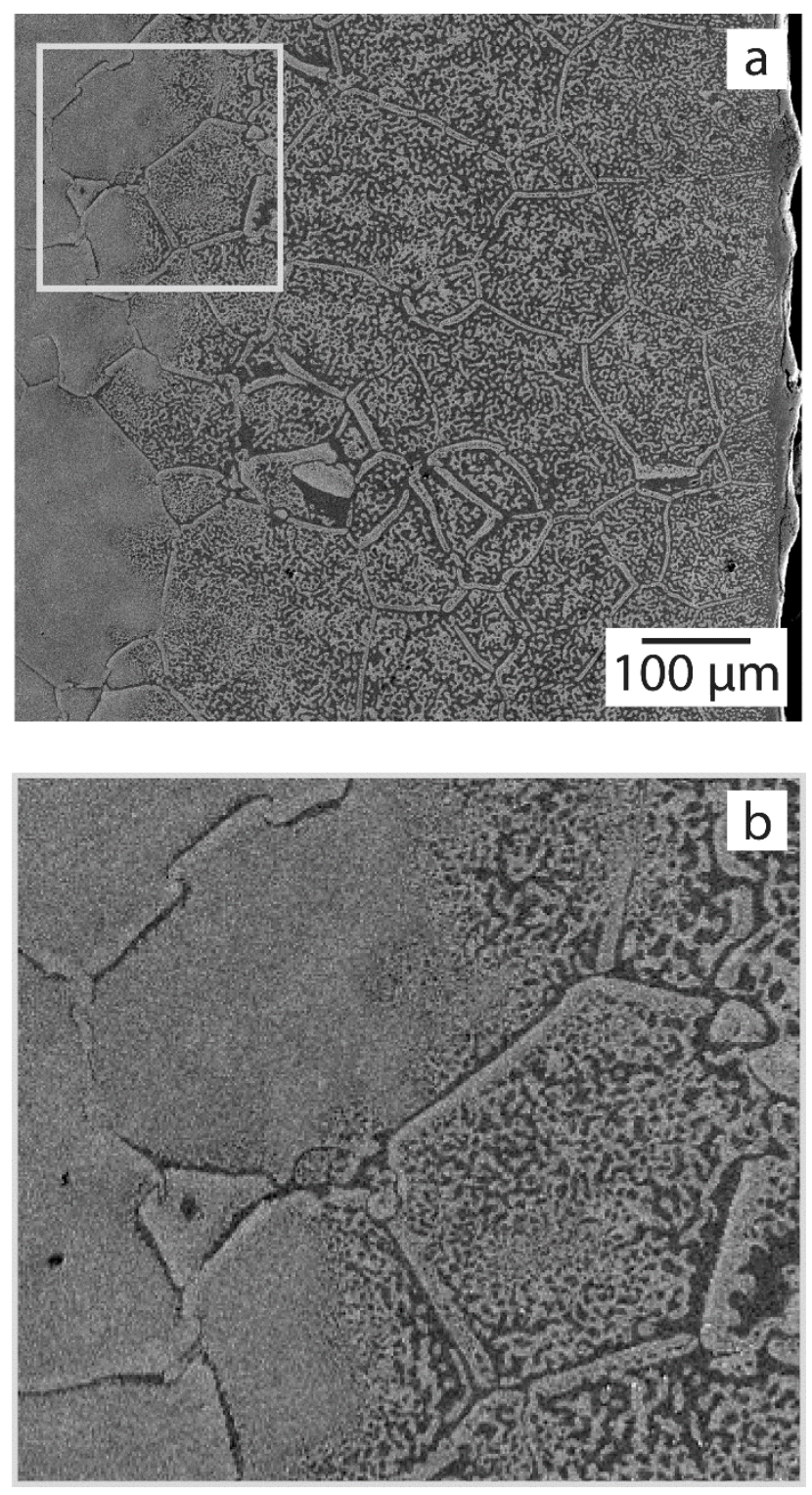

Fig. 8. Effect of grain boundaries on dealloying kinetics. Ti65Ta35 (dealloyed depth data not included in this work) sample dealloyed at $1160^{\circ} \mathrm{C}$ for 120 seconds. (a) The grains in this structure are small, causing an inhomogenous dealloying interface, contrasting with Fig. 1. (b) It can also been seen that the boundaries dissolve faster than the interior of the grains. 

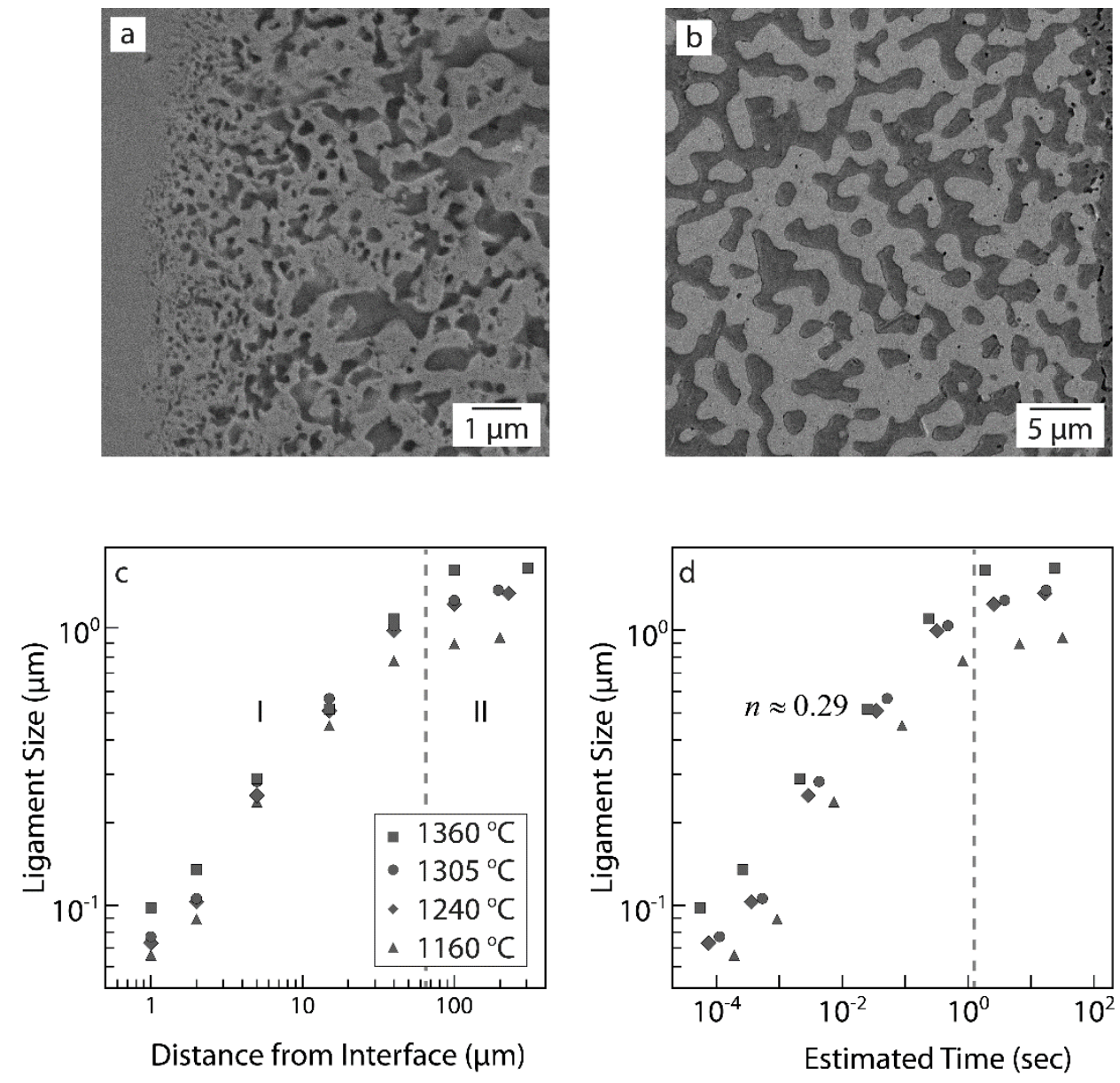

Fig. 9. Ligament coarsening during LMD using $\mathrm{Ti}_{40} \mathrm{Ta}_{60}$ as an example. (A) SEM micrograph of the dealloying interface (left of the image) showing ligaments $\sim 80 \mathrm{~nm}$ at the interface. (B) SEM micrograph of the edge of the sample (right of the image) showing $\sim 1.5 \mu \mathrm{m}$ ligaments at the edge of the sample. (C) Ligament size versus distance away from the dealloying interface. We see two distinct regions which are the result of different coarsening mechanisms dominating. (D) Ligament size versus estimated time using Eq. Error! Reference source not found. and values from Table 1. Using Eq. Error! Reference source not found. we can deconvolute the time-dependence of the dealloying depth and estimate the power-law exponent and activation energy as a function of time. 

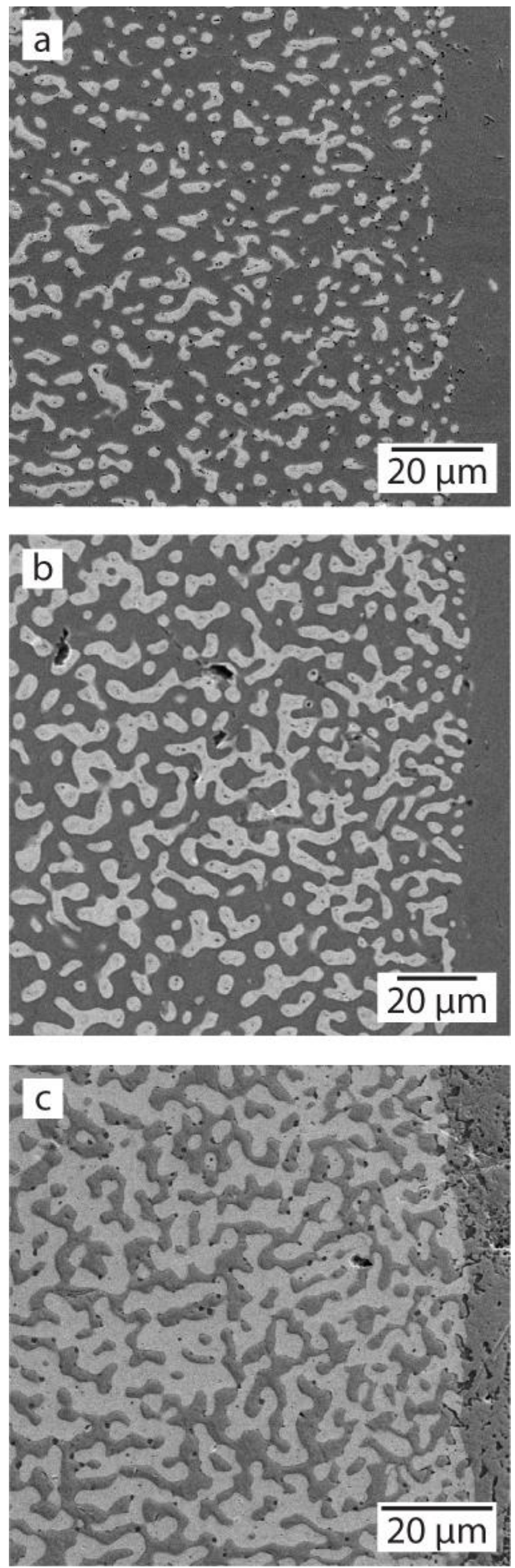

Fig. 10. Difference in morphology at the edge of samples dealloyed for 20 seconds at $1305{ }^{\circ} \mathrm{C}$ as a function of alloy composition (in all cases the morphology underwent significant coarsening): (A) $\mathrm{Ti}_{70} \mathrm{Ta}_{30}$ showing mostly disconnected blobs, (B) $\mathrm{Ti}_{55} \mathrm{Ta}_{45}$ showing a mixture of disconnected blobs and connected ligaments, and (C) $\mathrm{Ti}_{40} \mathrm{Ta}_{60}$ showing a highly connected structure. 

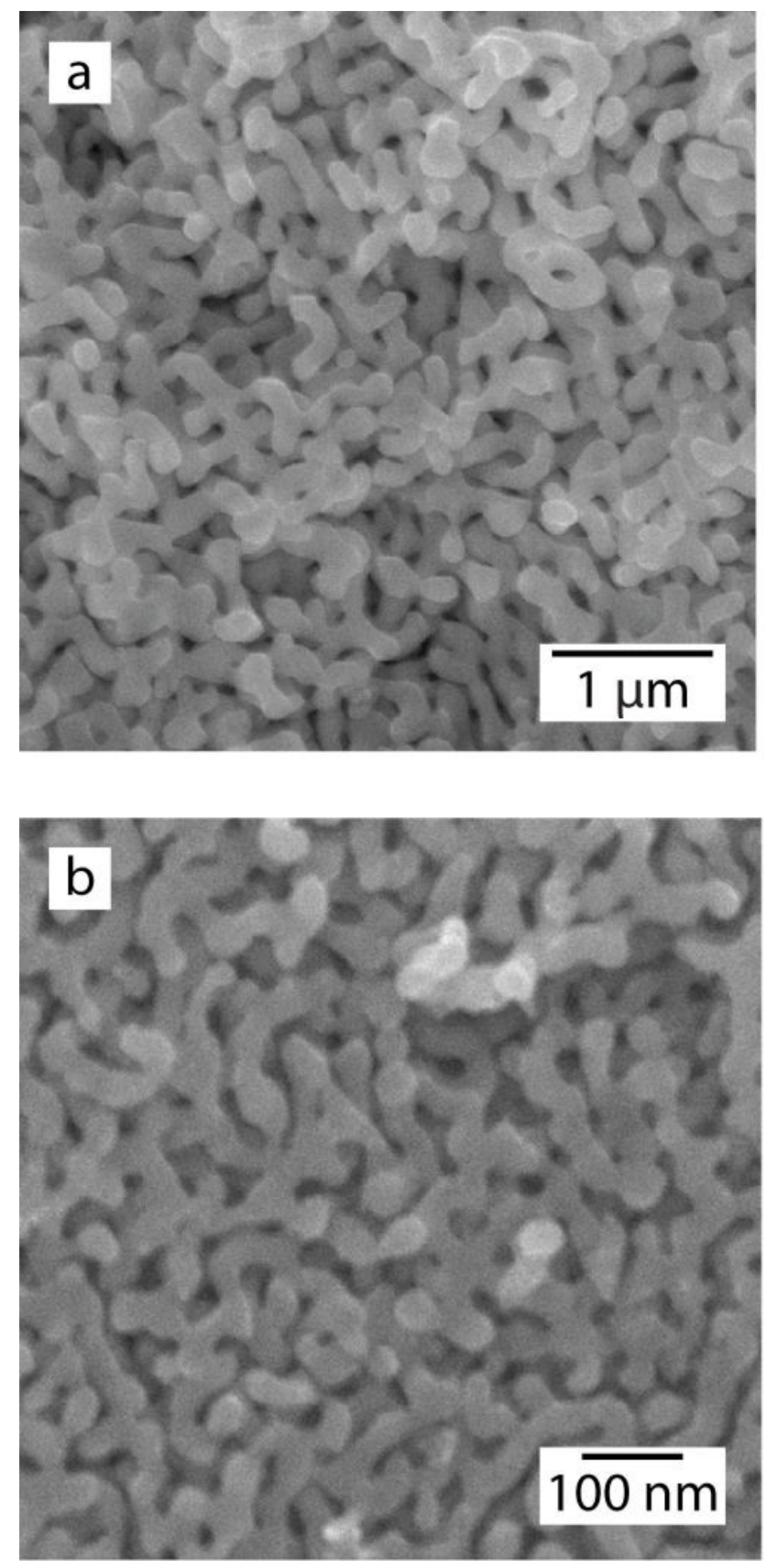

Fig. 11. Ligament size temperature dependence shown in porous Ta. (A) SEM micrograph of porous $\mathrm{Ta}$ with $\sim 100 \mathrm{~nm}$ ligaments, dealloyed for an hour in $\mathrm{Cu}_{32} \mathrm{Ag}_{68}\left(\mathrm{~T}_{\mathrm{m}} \sim 780{ }^{\circ} \mathrm{C}\right)$. (A) SEM micrograph of porous Ta with $\sim 50 \mathrm{~nm}$ ligaments, dealloyed for an hour in $\mathrm{Cu}_{20} \mathrm{Ag}_{40} \mathrm{Bi}_{40}\left(\mathrm{~T}_{\mathrm{m}} \sim 600\right.$ $\left.{ }^{\circ} \mathrm{C}\right)$. 
Table 1. Kinetic parameters determined for the compositions in this study.

\begin{tabular}{|c|c|c|c|c|c|}
\hline Composition & $D_{L}\left(1240{ }^{\circ} \mathrm{C}\right) \mathrm{cm}^{2} \mathrm{~s}^{-1}$ & $\Omega$ & $p$ & $D_{0}\left(\mathrm{~cm}^{2} \mathrm{~s}^{-1}\right)$ & $E_{a}(\mathrm{eV})$ \\
\hline $\begin{array}{l}\text { Literature } \\
\text { Value }\end{array}$ & $7.0 \times 10^{-5}$ & - & - & $1.7 \times 10^{-2}$ & 0.715 \\
\hline $\mathrm{Ti}_{70} \mathrm{Ta}_{30}$ & $6.5 \times 10^{-5}$ & 3.67 & 0.748 & $\begin{array}{l}2.4 \times 10^{-3} \\
4.5 \times 10^{-3, *} \\
4.2 \times 10^{-3,,^{*}}\end{array}$ & $\begin{array}{l}0.57 \\
0.63^{*} \\
0.38^{\text {** }}\end{array}$ \\
\hline $\mathrm{Ti}_{55} \mathrm{Ta}_{45}$ & $3.3 \times 10^{-5}$ & 2.44 & 0.855 & $\begin{array}{l}1.1 \times 10^{-3} \\
4.0 \times 10^{-3, *} \\
8.0 \times 10^{-4,{ }^{* *}}\end{array}$ & $\begin{array}{l}0.58 \\
0.74^{*} \\
0.56^{\text {** }}\end{array}$ \\
\hline $\mathrm{Ti}_{40} \mathrm{Ta}_{60}$ & $4.8 \times 10^{-5}$ & 4.33 & 1.019 & $\begin{array}{l}3.5 \times 10^{-2} \\
2.8 \times 10^{-2, *} \\
3.0 \times 10^{-2, * *}\end{array}$ & $\begin{array}{l}1.16 \\
1.12^{*} \\
1.16^{\text {** }}\end{array}$ \\
\hline
\end{tabular}

*Values calculated using data from the earliest dealloying times **Values calculated using data from the longest dealloying times 